Supporting Information for

\title{
Synthesis and Properties of Thiophene-fused Thiopyrylium Salts
}

\author{
Noriyoshi Nagahora, ${ }^{* \dagger}$ Kana Kitahara,${ }^{\dagger}$ Yoshiyuki Mizuhata, ${ }^{\S}$ Norihiro Tokitoh, ${ }^{\S}$ \\ Kosei Shioji, ${ }^{\dagger}$ and Kentaro Okuma ${ }^{\dagger}$ \\ ${ }^{\dagger}$ Department of Chemistry, Faculty of Science, Fukuoka University, Jonan-ku, Fukuoka \\ 814-0180, Japan \\ ${ }^{\S}$ Institute for Chemical Research, Kyoto University, Gokasho, Uji, Kyoto 611-0011 \\ E-mail: nagahora@fukuoka-u.ac.jp
}

Table of Contents

$\begin{array}{ll}\text { X-Ray Crystallographic Analysis } & \text { S2-S4 }\end{array}$

$\begin{array}{ll}\text { Theoretical Calculations } & \text { S5-S18 }\end{array}$

${ }^{1} \mathrm{H}$ and ${ }^{13} \mathrm{C}$ NMR Spectra of All New Compounds $\quad$ S19-S48

Thermogravimetric and Differential Thermal Analysis $\quad$ S49-S51

$\begin{array}{ll}\text { References } & \text { S52 }\end{array}$ 
X-Ray Crystallographic Analysis of Thiopyrylium Hexachlorostannate 1. Single crystals of thiopyrylium hexachlorostannate $\mathbf{1}$ were obtained by slow recrystallization from $\mathrm{CH} 3 \mathrm{CN}$ solution at $-20^{\circ} \mathrm{C}$. Intensity data were collected on a Rigaku Saturn CCD system with VariMax Mo Optics using Mo-Ka radiation $(\lambda=0.71075 \AA)$. Crystal data of $\mathbf{1}$ are provided in Table S1. The structure was solved by direct method and refined by full-matrix least-squares on $F^{2}$ for all reflections (SHELXL-2018). ${ }^{1}$ All non-hydrogen atoms were refined anisotropically. All hydrogen atoms were refined using a riding model. The crystallographic data reported in this manuscript have been deposited at the Cambridge Crystallographic Data Centre (CCDC) as supplementary publication no. CCDC-1972982. Copies of the data can be obtained free of charge via www.ccdc.cam.ac.uk/conts/retrieving.html (or from the Cambridge Crystallographic Data Centre, 12, Union Road, Cambridge, CB2 1EZ, UK; fax: +44 1223 336033; or deposit@ccdc.cam.ac.uk). 
Table S1. Crystallographic Data and Experimental Parameters for the Crystal Structure Analysis of 1.

\begin{tabular}{|c|c|}
\hline & 1 \\
\hline Formula & $2\left(\mathrm{C}_{19} \mathrm{H}_{17} \mathrm{~S}_{2}\right) \mathrm{Cl}_{6} \mathrm{Sn} 6\left(\mathrm{C}_{2} \mathrm{H}_{3} \mathrm{~N}\right)$ \\
\hline Formula weight & 1196.60 \\
\hline Crystal dimensions/mm & $0.21 \times 0.02 \times 0.01$ \\
\hline Collection temperature/K & $113(2)$ \\
\hline Wavelength $/ \AA$ & 0.71073 \\
\hline Crystal system & triclinic \\
\hline Space group & $P-1(\# 2)$ \\
\hline$a / \AA$ & $8.9661(8)$ \\
\hline$b / \AA$ & $12.1471(9)$ \\
\hline$c / \AA$ & $12.7912(13)$ \\
\hline$\alpha /^{\circ}$ & $91.881(7)$ \\
\hline$\beta /^{\circ}$ & $100.721(8)$ \\
\hline$\gamma^{\circ}$ & $93.595(7)$ \\
\hline Volume $/ \AA^{3}$ & $1364.7(2)$ \\
\hline$Z$ & 1 \\
\hline Density $/ \mathrm{gcm}^{-3}$ & 1.456 \\
\hline Reflections collected & 11365 \\
\hline Independent reflections & 4928 \\
\hline No. of parameters & 310 \\
\hline$R_{1}[I>2 \sigma(I)]$ & 0.0482 \\
\hline $\mathrm{w} R_{2}[I>2 \sigma(I)]$ & 0.1212 \\
\hline$R_{1}$ (all data) & 0.0730 \\
\hline $\mathrm{w} R_{2}$ (all data) & 0.1331 \\
\hline Goodness of fit & 1.028 \\
\hline Largest diff. peak/hole/e $\AA^{-3}$ & $1.773 /-0.963$ \\
\hline
\end{tabular}




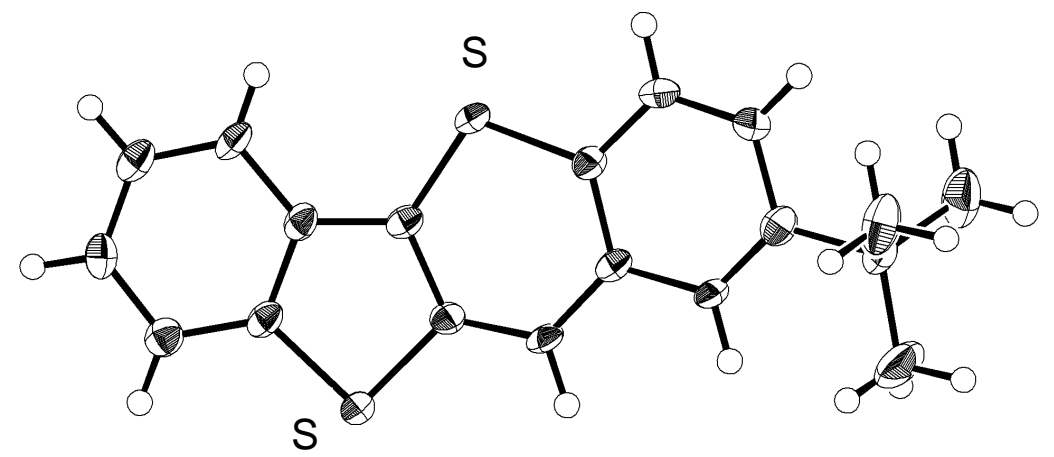

Figure S1. Molecular structure of 1 with thermal ellipsoids at $50 \%$ probability. Counter anion and solvate molecules and have been omitted for clarity.
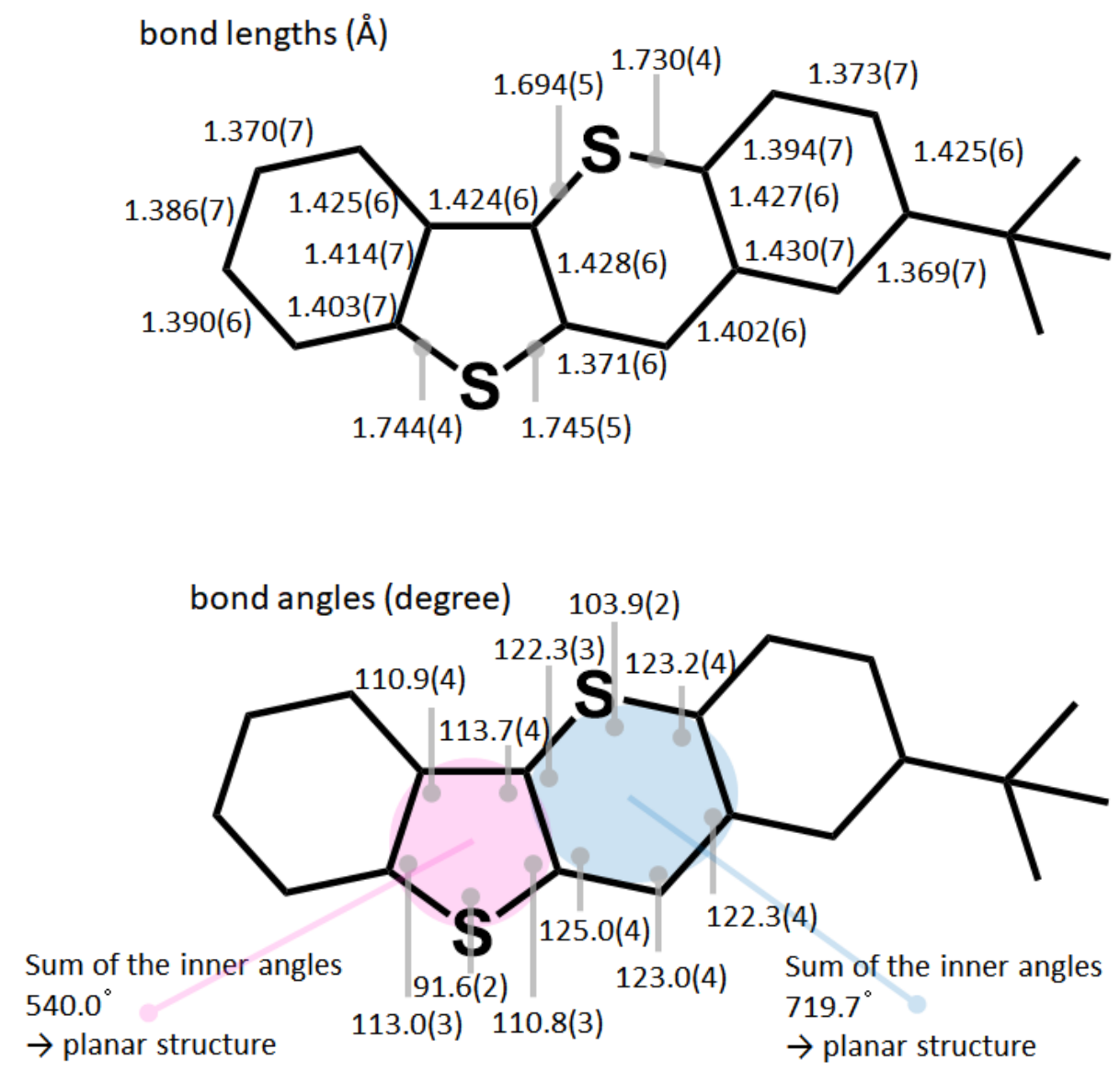

Figure S2. Selected bond lengths $[\AA]$ and angles $\left[{ }^{\circ}\right]$ of the solid state for $\mathbf{1}$. 
Theoretical Calculations. All theoretical calculations were carried out using the Gaussian 16 package program ${ }^{2}$ with density function theory at the B3LYP level. ${ }^{3}$ The geometries of $1^{\prime}-6^{\prime}$, where the counter anions were omitted, were optimized by the $6-311+\mathrm{G}(2 \mathrm{~d}, \mathrm{p})$ basis sets. All physico-chemical parameters (NMR, UV-vis, and NPA) were calculated at the B3LYP/6-311+G(2d,p) level of theory using the aforementioned optimized structures. NMR shielding tensors including nucleus-independent chemical shift (NICS) values were calculated using the gauge-independent atomic orbital (GIAO) method. UV-vis absorption wavelengths and oscillator strengths were obtained from time-dependent (TD) DFT calculations. NPA charges were calculated using NBO program implemented in the Gaussian 16 package. 
Table S2. Observed and calculated ${ }^{1} \mathrm{H}$ NMR chemical shifts [ppm] of $\mathbf{1}-\mathbf{6}^{a}$ and $\mathbf{1}^{\prime}-\mathbf{6}^{\bullet b}$.
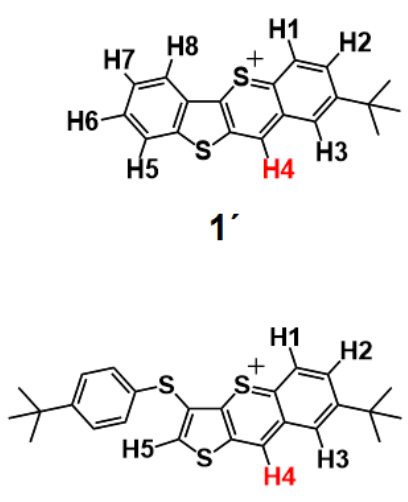

3'

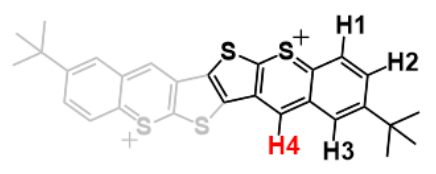

$5^{\prime}$
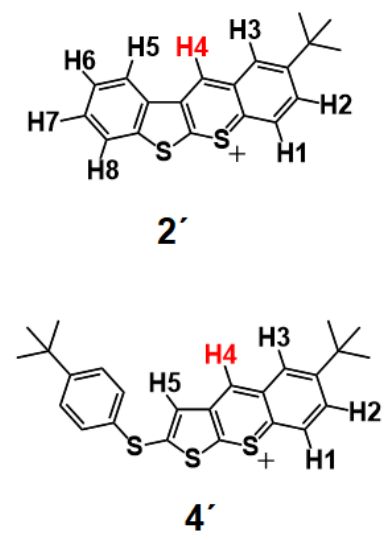

4

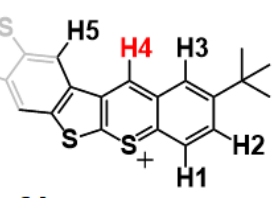

$6^{\prime}$

\begin{tabular}{llllllllll}
\hline Compd. & H1 & H2 & H3 & H4 & H5 & H6 & H7 & H8 & $\mathrm{CH}_{3}$ \\
\hline $\mathbf{1}$ & 8.74 & 8.48 & 8.67 & 9.98 & 8.36 & 8.07 & 7.87 & 8.74 & 1.53 \\
$\mathbf{1}^{\prime}$ (calcd) & 8.45 & 8.60 & 8.41 & 9.19 & 8.22 & 8.20 & 7.96 & 8.59 & 1.29 \\
$\mathbf{2}$ & 8.67 & 8.44 & 8.77 & 10.11 & 8.29 & 7.90 & 7.90 & 8.67 & 1.53 \\
$\mathbf{2}^{\prime}$ (calcd) & 8.36 & 8.48 & 8.76 & 9.40 & 8.58 & 8.05 & 8.10 & 8.17 & 1.33 \\
$\mathbf{3}$ & 8.65 & 8.47 & 8.68 & 10.00 & 9.30 & - & - & - & 1.50 \\
$\mathbf{3}^{\prime}$ (calcd) & 8.52 & 8.53 & 8.63 & 9.06 & 7.56 & - & - & - & 1.30 \\
$\mathbf{4}$ & 8.58 & 8.38 & 8.63 & 9.56 & 8.00 & - & - & - & 1.49 \\
$\mathbf{4}^{\prime}$ (calcd) & 8.17 & 8.33 & 8.32 & 8.70 & 7.25 & - & - & - & 1.22 \\
$\mathbf{5}^{\prime}$ & 8.82 & 8.59 & 8.86 & 10.35 & - & - & - & - & 1.55 \\
$\mathbf{5}^{\prime}$ (calcd) & 8.65 & 9.02 & 8.78 & 9.36 & - & - & - & - & 1.40 \\
$\mathbf{6}^{\prime}$ (calcd) & 8.73 & 8.51 & 8.88 & 10.28 & 9.52 & - & - & - & 1.55 \\
$\mathbf{6}^{\prime}$ (calc) & 8.81 & 8.98 & 9.55 & 9.04 & - & - & - & 1.42 \\
\hline
\end{tabular}

${ }^{a}$ The experimentally observed chemical shifts were recorded in $\mathrm{CD}_{3} \mathrm{CN}$.

${ }^{b}$ Calculations were carried out at the GIAO-B3LYP/6-311+G(2d,p)//B3LYP/6-311+G(2d,p) level of theory. 
Figure S3. Summary of the Natural Population Analysis of Thiopyrylium Cations $\mathbf{1}^{\prime}-\mathbf{6}^{\prime}$.
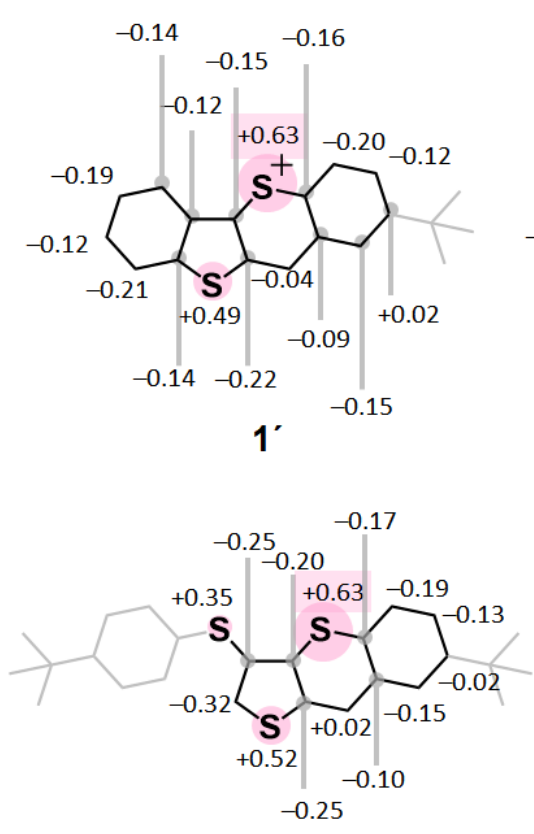

3

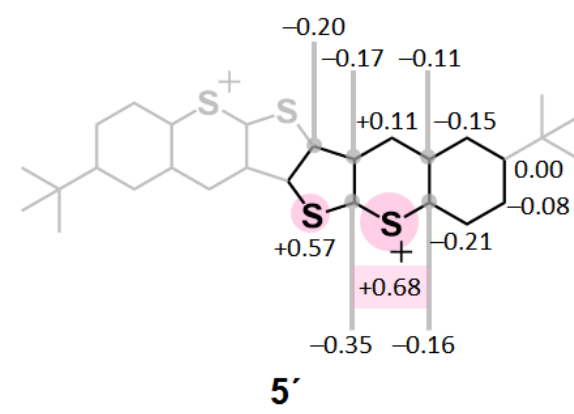

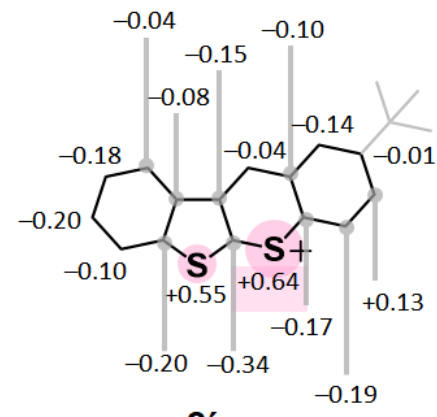

2

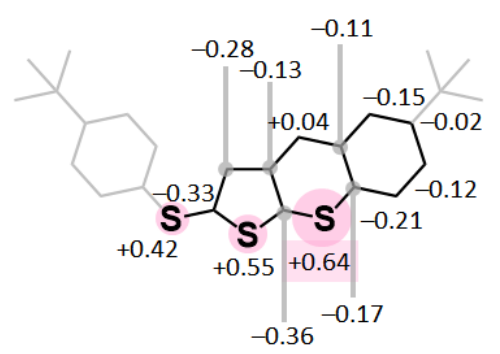

$4^{\prime}$

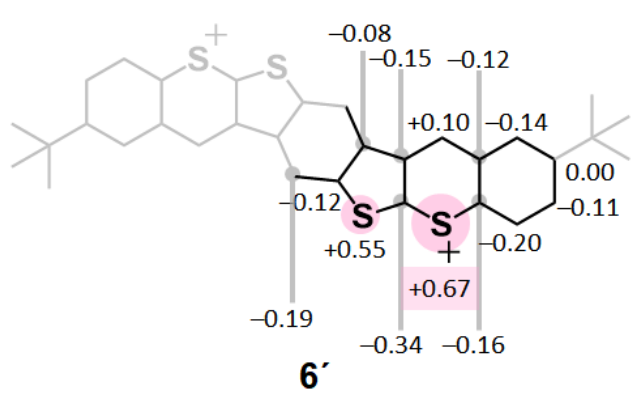


Table S3. Theoretically Optimized Cartesian Coordinates of Thiopyrylium Cation 1' at B3LYP/6-311+G(2d,p) Level of Theory

\begin{tabular}{|c|c|c|c|}
\hline atom & $\mathrm{X}$ & $\mathrm{y}$ & $\mathrm{Z}$ \\
\hline $\mathrm{C}$ & 1.209499 & 0.53661 & 0 \\
\hline $\mathrm{C}$ & -0.09037 & 1.109431 & 0 \\
\hline $\mathrm{C}$ & -0.20507 & 2.523958 & 0 \\
\hline $\mathrm{C}$ & 0.893545 & 3.355181 & 0 \\
\hline $\mathrm{C}$ & 2.170417 & 2.736302 & 0 \\
\hline $\mathrm{C}$ & 2.334005 & 1.370018 & 0 \\
\hline S & 1.494425 & -1.17618 & 0 \\
\hline $\mathrm{C}$ & -0.06963 & -1.85857 & 0 \\
\hline $\mathrm{C}$ & -1.24248 & -1.06637 & 0 \\
\hline $\mathrm{C}$ & -1.25365 & 0.309553 & 0 \\
\hline $\mathrm{C}$ & 0.793534 & 4.883987 & 0 \\
\hline $\mathrm{C}$ & -0.6646 & 5.368831 & 0 \\
\hline $\mathrm{C}$ & 1.494425 & 5.433851 & 1.263445 \\
\hline $\mathrm{C}$ & 1.494425 & 5.433851 & -1.26345 \\
\hline $\mathrm{C}$ & -0.33158 & -3.25971 & 0 \\
\hline $\mathrm{C}$ & -1.72347 & -3.52043 & 0 \\
\hline S & -2.69312 & -2.06188 & 0 \\
\hline $\mathrm{C}$ & 0.57644 & -4.33626 & 0 \\
\hline $\mathrm{C}$ & 0.089565 & -5.62364 & 0 \\
\hline $\mathrm{C}$ & -1.29662 & -5.86446 & 0 \\
\hline $\mathrm{C}$ & -2.21053 & -4.82472 & 0 \\
\hline $\mathrm{H}$ & -1.20464 & 2.936478 & 0 \\
\hline $\mathrm{H}$ & 3.059218 & 3.353569 & 0 \\
\hline $\mathrm{H}$ & 3.32935 & 0.942266 & 0 \\
\hline $\mathrm{H}$ & -2.20939 & 0.822623 & 0 \\
\hline $\mathrm{H}$ & -0.68316 & 6.459476 & 0 \\
\hline $\mathrm{H}$ & -1.20803 & 5.036376 & -0.88778 \\
\hline $\mathrm{H}$ & -1.20803 & 5.036376 & 0.887781 \\
\hline $\mathrm{H}$ & 1.434695 & 6.523821 & 1.272098 \\
\hline $\mathrm{H}$ & 2.551564 & 5.164612 & 1.301152 \\
\hline $\mathrm{H}$ & 1.017737 & 5.063026 & 2.173488 \\
\hline $\mathrm{H}$ & 1.017737 & 5.063026 & -2.17349 \\
\hline $\mathrm{H}$ & 1.434695 & 6.523821 & -1.2721 \\
\hline $\mathrm{H}$ & 2.551564 & 5.164612 & -1.30115 \\
\hline $\mathrm{H}$ & 1.644696 & -4.15599 & 0 \\
\hline $\mathrm{H}$ & 0.775642 & -6.46041 & 0 \\
\hline $\mathrm{H}$ & -1.65744 & -6.88545 & 0 \\
\hline $\mathrm{H}$ & -3.27432 & -5.0237 & 0 \\
\hline
\end{tabular}


Table S4. Theoretically Optimized Cartesian Coordinates of Thiopyrylium Cation 2' at B3LYP/6-311+G(2d,p) Level of Theory

\begin{tabular}{llll}
\hline atom & $\mathrm{x}$ & $\mathrm{y}$ & $\mathrm{z}$ \\
\hline $\mathrm{C}$ & 1.204287 & 1.006514 & 0 \\
$\mathrm{C}$ & 1.884877 & 2.29439 & 0 \\
$\mathrm{C}$ & 3.280394 & 2.154846 & 0 \\
$\mathrm{~S}$ & 3.779962 & 0.454853 & 0 \\
$\mathrm{C}$ & 2.138359 & -0.06448 & 0 \\
$\mathrm{~S}$ & 1.731293 & -1.71483 & 0 \\
$\mathrm{C}$ & -0.00933 & -1.69661 & 0 \\
$\mathrm{C}$ & -0.76985 & -0.4961 & 0 \\
$\mathrm{C}$ & -0.15634 & 0.776796 & 0 \\
$\mathrm{C}$ & 1.338066 & 3.580607 & 0 \\
$\mathrm{C}$ & 2.184427 & 4.677987 & 0 \\
$\mathrm{C}$ & 3.572782 & 4.512493 & 0 \\
$\mathrm{C}$ & 4.139564 & 3.244766 & 0 \\
$\mathrm{C}$ & -0.65819 & -2.93115 & 0 \\
$\mathrm{C}$ & -2.03883 & -2.98053 & 0 \\
$\mathrm{C}$ & -2.84017 & -1.81679 & 0 \\
$\mathrm{C}$ & -2.18022 & -0.60187 & 0 \\
$\mathrm{C}$ & -4.37528 & -1.87061 & 0 \\
$\mathrm{C}$ & -4.90545 & -3.31389 & 0 \\
$\mathrm{C}$ & -4.90545 & -1.15501 & 1.262978 \\
$\mathrm{C}$ & -4.90545 & -1.15501 & -1.26298 \\
$\mathrm{H}$ & -0.81775 & 1.636295 & 0 \\
$\mathrm{H}$ & 0.264941 & 3.727087 & 0 \\
$\mathrm{H}$ & 1.765808 & 5.676068 & 0 \\
$\mathrm{H}$ & 4.217396 & 5.382146 & 0 \\
$\mathrm{H}$ & 5.214385 & 3.117761 & 0 \\
$\mathrm{H}$ & -0.0852 & -3.85067 & 0 \\
$\mathrm{H}$ & -2.50822 & -3.95388 & 0 \\
$\mathrm{H}$ & -2.74426 & 0.322567 & 0 \\
$\mathrm{H}$ & -5.99611 & -3.29664 & 0 \\
$\mathrm{H}$ & -4.59136 & -3.8686 & -0.88734 \\
$\mathrm{H}$ & -4.59136 & -3.8686 & 0.887343 \\
$\mathrm{H}$ & -5.99654 & -1.18776 & 1.275477 \\
$\mathrm{H}$ & -4.60839 & -0.10503 & 1.296275 \\
$\mathrm{H}$ & -4.54391 & -1.63824 & 2.173316 \\
$\mathrm{H}$ & -4.54391 & -1.63824 & -2.17332 \\
$\mathrm{H}$ & -5.99654 & -1.18776 & -1.27548 \\
\hline & -4.60839 & -0.10503 & -1.29628 \\
\hline & & &
\end{tabular}


Table S5. Theoretically Optimized Cartesian Coordinates of Thiopyrylium Cation $3^{\prime}$ at B3LYP/6-311+G(2d,p) Level of Theory

\begin{tabular}{llll}
\hline atom & $\mathrm{x}$ & $\mathrm{y}$ & $\mathrm{z}$ \\
\hline $\mathrm{C}$ & 3.225313 & -0.90955 & -0.00162 \\
$\mathrm{C}$ & 3.568402 & 0.46956 & 0.002299 \\
$\mathrm{C}$ & 2.581978 & 1.480707 & 0.010117 \\
$\mathrm{C}$ & 1.232734 & 1.223988 & 0.014855 \\
$\mathrm{C}$ & 0.65159 & -0.06639 & 0.012199 \\
$\mathrm{~S}$ & 1.589556 & -1.49934 & 0.002339 \\
$\mathrm{~S}$ & -0.01697 & 2.45404 & 0.024986 \\
$\mathrm{C}$ & -1.23745 & 1.24017 & 0.025903 \\
$\mathrm{C}$ & -0.76774 & -0.05141 & 0.018664 \\
$\mathrm{C}$ & 4.243015 & -1.87097 & -0.00904 \\
$\mathrm{C}$ & 5.560719 & -1.47441 & -0.01244 \\
$\mathrm{C}$ & 5.950921 & -0.11063 & -0.00883 \\
$\mathrm{C}$ & 4.942047 & 0.827033 & -0.00159 \\
$\mathrm{~S}$ & -1.71195 & -1.54732 & 0.018123 \\
$\mathrm{C}$ & -3.4005 & -0.94892 & 0.019162 \\
$\mathrm{C}$ & -4.07437 & -0.76661 & 1.222089 \\
$\mathrm{C}$ & -5.40539 & -0.35942 & 1.215456 \\
$\mathrm{C}$ & -6.09712 & -0.13237 & 0.02223 \\
$\mathrm{C}$ & -5.39448 & -0.3321 & -1.17533 \\
$\mathrm{C}$ & -4.06864 & -0.73806 & -1.18722 \\
$\mathrm{C}$ & -7.56828 & 0.30486 & -0.01617 \\
$\mathrm{C}$ & -8.1647 & 0.473148 & 1.390753 \\
$\mathrm{C}$ & -7.67878 & 1.657425 & -0.75512 \\
$\mathrm{C}$ & -8.39475 & -0.76191 & -0.76937 \\
$\mathrm{C}$ & 7.439639 & 0.251929 & -0.0129 \\
$\mathrm{C}$ & 8.107075 & -0.34707 & 1.246168 \\
$\mathrm{C}$ & 8.098326 & -0.3389 & -1.28039 \\
$\mathrm{C}$ & 7.665045 & 1.77192 & -0.0088 \\
$\mathrm{H}$ & 2.92043 & 2.511257 & 0.012598 \\
$\mathrm{H}$ & -2.27619 & 1.534081 & 0.031985 \\
$\mathrm{H}$ & 3.993932 & -2.92533 & -0.01201 \\
$\mathrm{H}$ & 6.321598 & -2.244 & -0.01806 \\
$\mathrm{H}$ & 5.175138 & 1.88303 & 0.001482 \\
$\mathrm{H}$ & -3.56923 & -0.95025 & 2.162194 \\
$\mathrm{H}$ & -5.9023 & -0.22963 & 2.166333 \\
\hline & & & \\
$\mathrm{H}$ & & & \\
$\mathrm{H}$ & & &
\end{tabular}




\begin{tabular}{llll}
\hline $\mathrm{H}$ & -5.89135 & -0.17869 & -2.12478 \\
$\mathrm{H}$ & -3.55757 & -0.90069 & -2.12796 \\
$\mathrm{H}$ & -9.20699 & 0.785994 & 1.30804 \\
$\mathrm{H}$ & -8.14938 & -0.46101 & 1.95675 \\
$\mathrm{H}$ & -7.64022 & 1.236812 & 1.970006 \\
$\mathrm{H}$ & -8.72246 & 1.977205 & -0.79325 \\
$\mathrm{H}$ & -7.31548 & 1.594592 & -1.78234 \\
$\mathrm{H}$ & -7.10752 & 2.434303 & -0.24131 \\
$\mathrm{H}$ & -8.33488 & -1.73097 & -0.26903 \\
$\mathrm{H}$ & -9.44498 & -0.46377 & -0.80355 \\
$\mathrm{H}$ & -8.05488 & -0.89295 & -1.7981 \\
$\mathrm{H}$ & 9.170497 & -0.10031 & 1.251878 \\
$\mathrm{H}$ & 7.66268 & 0.055653 & 2.159008 \\
$\mathrm{H}$ & 8.024092 & -1.43487 & 1.280907 \\
$\mathrm{H}$ & 9.16164 & -0.0919 & -1.29196 \\
$\mathrm{H}$ & 7.647504 & 0.069544 & -2.18752 \\
$\mathrm{H}$ & 8.015311 & -1.42648 & -1.32149 \\
$\mathrm{H}$ & 7.246577 & 2.246722 & 0.881979 \\
$\mathrm{H}$ & 8.736042 & 1.979052 & -0.012 \\
$\mathrm{H}$ & 7.240133 & 2.252436 & -0.89344 \\
\hline
\end{tabular}


Table S6. Theoretically Optimized Cartesian Coordinates of Thiopyrylium Cation 4' at B3LYP/6-311+G(2d,p) Level of Theory

\begin{tabular}{|c|c|c|c|}
\hline atom & $\mathrm{x}$ & $\mathrm{y}$ & $\mathrm{Z}$ \\
\hline $\mathrm{C}$ & -3.03316 & 0.02452 & -0.00453 \\
\hline $\mathrm{C}$ & -3.732 & -1.21284 & 0.011594 \\
\hline S & -2.93219 & -2.75294 & 0.020634 \\
\hline $\mathrm{C}$ & -1.28764 & -2.33484 & 0.00686 \\
\hline $\mathrm{C}$ & -0.77741 & -1.0089 & -0.00868 \\
\hline $\mathrm{C}$ & -1.62603 & 0.088963 & -0.01391 \\
\hline S & -0.03275 & -3.51211 & 0.010598 \\
\hline $\mathrm{C}$ & 1.209022 & -2.23698 & -0.0097 \\
\hline $\mathrm{C}$ & 0.656674 & -0.9976 & -0.01779 \\
\hline $\mathrm{C}$ & -3.79794 & 1.222803 & -0.01084 \\
\hline $\mathrm{C}$ & -5.17443 & 1.225309 & -0.00166 \\
\hline $\mathrm{C}$ & -5.82387 & -0.03705 & 0.014649 \\
\hline $\mathrm{C}$ & -5.13223 & -1.22559 & 0.021097 \\
\hline$S$ & 2.868575 & -2.79103 & -0.01498 \\
\hline $\mathrm{C}$ & 3.778431 & -1.24776 & -0.01967 \\
\hline $\mathrm{C}$ & 4.183541 & -0.68408 & -1.22445 \\
\hline $\mathrm{C}$ & 4.95349 & 0.475261 & -1.22 \\
\hline $\mathrm{C}$ & 5.341666 & 1.093412 & -0.02791 \\
\hline $\mathrm{C}$ & 4.922083 & 0.498374 & 1.171199 \\
\hline $\mathrm{C}$ & 4.15563 & -0.65675 & 1.186011 \\
\hline $\mathrm{C}$ & 6.203907 & 2.363222 & 0.007934 \\
\hline $\mathrm{C}$ & 6.562583 & 2.866218 & -1.39984 \\
\hline $\mathrm{C}$ & 5.431918 & 3.483059 & 0.74118 \\
\hline $\mathrm{C}$ & 7.517217 & 2.063273 & 0.765351 \\
\hline $\mathrm{C}$ & -6.01454 & 2.506863 & -0.00794 \\
\hline $\mathrm{C}$ & -5.14198 & 3.771571 & -0.02735 \\
\hline C & -6.91521 & 2.512808 & -1.26424 \\
\hline $\mathrm{C}$ & -6.89541 & 2.537815 & 1.261966 \\
\hline $\mathrm{H}$ & -1.17504 & 1.075461 & -0.02602 \\
\hline $\mathrm{H}$ & 1.242388 & -0.09002 & -0.03007 \\
\hline $\mathrm{H}$ & -3.24822 & 2.153879 & -0.02326 \\
\hline $\mathrm{H}$ & -6.9051 & -0.07884 & 0.022429 \\
\hline $\mathrm{H}$ & -5.66895 & -2.16667 & 0.033526 \\
\hline $\mathrm{H}$ & 3.911503 & -1.15016 & -2.16326 \\
\hline
\end{tabular}




\begin{tabular}{llll}
\hline $\mathrm{H}$ & 5.258147 & 0.88697 & -2.17164 \\
$\mathrm{H}$ & 5.207472 & 0.935371 & 2.119587 \\
$\mathrm{H}$ & 3.861725 & -1.10359 & 2.127556 \\
$\mathrm{H}$ & 7.17113 & 3.768578 & -1.31886 \\
$\mathrm{H}$ & 7.143551 & 2.131412 & -1.96165 \\
$\mathrm{H}$ & 5.674587 & 3.122889 & -1.98249 \\
$\mathrm{H}$ & 6.037872 & 4.391057 & 0.778599 \\
$\mathrm{H}$ & 5.187068 & 3.207457 & 1.768427 \\
$\mathrm{H}$ & 4.499655 & 3.721667 & 0.22342 \\
$\mathrm{H}$ & 8.085256 & 1.273298 & 0.269223 \\
$\mathrm{H}$ & 8.141688 & 2.958861 & 0.797678 \\
$\mathrm{H}$ & 7.334672 & 1.750253 & 1.794729 \\
$\mathrm{H}$ & -5.78352 & 4.653855 & -0.03137 \\
$\mathrm{H}$ & -4.50097 & 3.841592 & 0.854804 \\
$\mathrm{H}$ & -4.51443 & 3.823613 & -0.92036 \\
$\mathrm{H}$ & -7.52286 & 3.419693 & -1.2775 \\
$\mathrm{H}$ & -7.59826 & 1.661784 & -1.28807 \\
$\mathrm{H}$ & -6.31841 & 2.493472 & -2.1788 \\
$\mathrm{H}$ & -6.28434 & 2.536822 & 2.167266 \\
$\mathrm{H}$ & -7.50321 & 3.44469 & 1.266643 \\
$\mathrm{H}$ & -7.57754 & 1.687272 & 1.313441 \\
\hline
\end{tabular}


Table S7. Theoretically Optimized Cartesian Coordinates of Thiopyrylium Cation $\mathbf{5}^{\mathbf{\prime}}$ at B3LYP/6-311+G(2d,p) Level of Theory

\begin{tabular}{llll}
\hline atom & $\mathrm{x}$ & $\mathrm{y}$ & $\mathrm{z}$ \\
\hline $\mathrm{C}$ & 1.521519 & 1.726985 & 0 \\
$\mathrm{C}$ & 0.107555 & 1.827409 & 0 \\
$\mathrm{C}$ & -0.45835 & 0.50586 & 0 \\
$\mathrm{C}$ & 0.458345 & -0.50586 & 0 \\
$\mathrm{~S}$ & 2.108241 & 0.089618 & 0 \\
$\mathrm{~S}$ & -2.10824 & -0.08962 & 0 \\
$\mathrm{C}$ & -1.52152 & -1.72699 & 0 \\
$\mathrm{C}$ & -0.10756 & -1.82741 & 0 \\
$\mathrm{~S}$ & -2.58737 & -3.04935 & 0 \\
$\mathrm{C}$ & -1.51893 & -4.41706 & 0 \\
$\mathrm{C}$ & -0.09469 & -4.30223 & 0 \\
$\mathrm{C}$ & 0.546289 & -3.05537 & 0 \\
$\mathrm{~S}$ & 2.587368 & 3.049351 & 0 \\
$\mathrm{C}$ & 1.518933 & 4.417062 & 0 \\
$\mathrm{C}$ & 0.094692 & 4.302229 & 0 \\
$\mathrm{C}$ & -0.54629 & 3.055367 & 0 \\
$\mathrm{C}$ & -2.1143 & -5.6802 & 0 \\
$\mathrm{C}$ & -1.31757 & -6.80487 & 0 \\
$\mathrm{C}$ & 0.102719 & -6.74839 & 0 \\
$\mathrm{C}$ & 0.676948 & -5.49647 & 0 \\
$\mathrm{C}$ & 2.114299 & 5.680201 & 0 \\
$\mathrm{C}$ & 1.317574 & 6.80487 & 0 \\
$\mathrm{C}$ & -0.10272 & 6.748392 & 0 \\
$\mathrm{C}$ & -0.67695 & 5.496473 & 0 \\
$\mathrm{C}$ & -0.90987 & 8.048011 & 0 \\
$\mathrm{C}$ & -2.42377 & 7.786801 & 0 \\
$\mathrm{C}$ & -0.54629 & 8.861113 & 1.264854 \\
$\mathrm{C}$ & -0.54629 & 8.861113 & -1.26485 \\
$\mathrm{C}$ & 0.909872 & -8.04801 & 0 \\
$\mathrm{C}$ & 0.546289 & -8.86111 & -1.26485 \\
$\mathrm{C}$ & 2.423767 & -7.7868 & 0 \\
$\mathrm{C}$ & 0.546289 & -8.86111 & 1.264854 \\
$\mathrm{H}$ & 1.631378 & -3.05976 & 0 \\
$\mathrm{H}$ & -1.63138 & 3.059755 & 0 \\
\hline & & & \\
& & & \\
& & &
\end{tabular}




\begin{tabular}{llll}
\hline $\mathrm{H}$ & -3.19312 & -5.78004 & 0 \\
$\mathrm{H}$ & -1.8073 & -7.76991 & 0 \\
$\mathrm{H}$ & 1.753026 & -5.38897 & 0 \\
$\mathrm{H}$ & 3.193121 & 5.780036 & 0 \\
$\mathrm{H}$ & 1.807299 & 7.76991 & 0 \\
$\mathrm{H}$ & -1.75303 & 5.388967 & 0 \\
$\mathrm{H}$ & -2.95392 & 8.739553 & 0 \\
$\mathrm{H}$ & -2.74737 & 7.239511 & -0.88898 \\
$\mathrm{H}$ & -2.74737 & 7.239511 & 0.888978 \\
$\mathrm{H}$ & -1.11255 & 9.793814 & 1.271913 \\
$\mathrm{H}$ & 0.512336 & 9.12472 & 1.301317 \\
$\mathrm{H}$ & -0.79251 & 8.311579 & 2.175967 \\
$\mathrm{H}$ & -0.79251 & 8.311579 & -2.17597 \\
$\mathrm{H}$ & -1.11255 & 9.793814 & -1.27191 \\
$\mathrm{H}$ & 0.512336 & 9.12472 & -1.30132 \\
$\mathrm{H}$ & 1.112547 & -9.79381 & -1.27191 \\
$\mathrm{H}$ & -0.51234 & -9.12472 & -1.30132 \\
$\mathrm{H}$ & 0.792511 & -8.31158 & -2.17597 \\
$\mathrm{H}$ & 2.953917 & -8.73955 & 0 \\
$\mathrm{H}$ & 2.747372 & -7.23951 & 0.888978 \\
$\mathrm{H}$ & 2.747372 & -7.23951 & -0.88898 \\
$\mathrm{H}$ & -0.51234 & -9.12472 & 1.301317 \\
$\mathrm{H}$ & 1.112547 & -9.79381 & 1.271913 \\
$\mathrm{H}$ & 0.792511 & -8.31158 & 2.175967 \\
\hline & & & \\
\hline & & & \\
& & &
\end{tabular}


Table S8. Theoretically Optimized Cartesian Coordinates of Thiopyrylium Cation 6' at B3LYP/6-311+G(2d,p) Level of Theory

\begin{tabular}{|c|c|c|c|}
\hline atom & $\mathrm{x}$ & $\mathrm{y}$ & $\mathrm{Z}$ \\
\hline $\mathrm{C}$ & 0.309785 & 1.329225 & 0 \\
\hline $\mathrm{C}$ & 1.350094 & 0.376155 & 0 \\
\hline $\mathrm{C}$ & 1.030809 & -0.98128 & 0 \\
\hline $\mathrm{C}$ & -0.30979 & -1.32923 & 0 \\
\hline $\mathrm{C}$ & -1.35009 & -0.37616 & 0 \\
\hline $\mathrm{C}$ & -1.03081 & 0.981278 & 0 \\
\hline S & -0.92306 & -2.98673 & 0 \\
\hline $\mathrm{C}$ & -2.55612 & -2.41481 & 0 \\
\hline $\mathrm{C}$ & -2.66688 & -1.0037 & 0 \\
\hline$S$ & 0.923058 & 2.986726 & 0 \\
\hline $\mathrm{C}$ & 2.556116 & 2.414808 & 0 \\
\hline $\mathrm{C}$ & 2.666883 & 1.003698 & 0 \\
\hline$S$ & -3.86252 & -3.499 & 0 \\
\hline $\mathrm{C}$ & -5.24962 & -2.44844 & 0 \\
\hline $\mathrm{C}$ & -5.14668 & -1.02639 & 0 \\
\hline $\mathrm{C}$ & -3.89912 & -0.37242 & 0 \\
\hline$S$ & 3.862521 & 3.499002 & 0 \\
\hline $\mathrm{C}$ & 5.249619 & 2.448438 & 0 \\
\hline $\mathrm{C}$ & 5.146684 & 1.026394 & 0 \\
\hline $\mathrm{C}$ & 3.899119 & 0.372422 & 0 \\
\hline $\mathrm{C}$ & 6.503621 & 3.054346 & 0 \\
\hline $\mathrm{C}$ & 7.642159 & 2.269418 & 0 \\
\hline $\mathrm{C}$ & 7.596804 & 0.855732 & 0 \\
\hline $\mathrm{C}$ & 6.343627 & 0.27108 & 0 \\
\hline $\mathrm{C}$ & -6.50362 & -3.05435 & 0 \\
\hline $\mathrm{C}$ & -7.64216 & -2.26942 & 0 \\
\hline $\mathrm{C}$ & -7.5968 & -0.85573 & 0 \\
\hline $\mathrm{C}$ & -6.34363 & -0.27108 & 0 \\
\hline $\mathrm{C}$ & -8.86456 & 0.009725 & 0 \\
\hline $\mathrm{C}$ & -10.1465 & -0.83929 & 0 \\
\hline $\mathrm{C}$ & -8.86456 & 0.900028 & 1.263865 \\
\hline $\mathrm{C}$ & -8.86456 & 0.900028 & -1.26387 \\
\hline $\mathrm{C}$ & 8.864558 & -0.00973 & 0 \\
\hline $\mathrm{C}$ & 10.14648 & 0.839285 & 0 \\
\hline
\end{tabular}




\begin{tabular}{|c|c|c|c|}
\hline $\mathrm{C}$ & 8.864558 & -0.90003 & 1.263865 \\
\hline $\mathrm{C}$ & 8.864558 & -0.90003 & -1.26387 \\
\hline $\mathrm{H}$ & 1.80166 & -1.74122 & 0 \\
\hline $\mathrm{H}$ & -1.80166 & 1.741224 & 0 \\
\hline $\mathrm{H}$ & -3.92033 & 0.711994 & 0 \\
\hline $\mathrm{H}$ & 3.92033 & -0.71199 & 0 \\
\hline $\mathrm{H}$ & 6.593169 & 4.134094 & 0 \\
\hline $\mathrm{H}$ & 8.599151 & 2.771642 & 0 \\
\hline $\mathrm{H}$ & 6.24872 & -0.80761 & 0 \\
\hline $\mathrm{H}$ & -6.59317 & -4.13409 & 0 \\
\hline $\mathrm{H}$ & -8.59915 & -2.77164 & 0 \\
\hline $\mathrm{H}$ & -6.24872 & 0.80761 & 0 \\
\hline $\mathrm{H}$ & -11.0141 & -0.1788 & 0 \\
\hline $\mathrm{H}$ & -10.2241 & -1.47129 & -0.88787 \\
\hline $\mathrm{H}$ & -10.2241 & -1.47129 & 0.887867 \\
\hline $\mathrm{H}$ & -9.76301 & 1.519315 & 1.275875 \\
\hline $\mathrm{H}$ & -8.00412 & 1.571561 & 1.295861 \\
\hline $\mathrm{H}$ & -8.86045 & 0.297525 & 2.174754 \\
\hline $\mathrm{H}$ & -8.86045 & 0.297525 & -2.17475 \\
\hline $\mathrm{H}$ & -9.76301 & 1.519315 & -1.27588 \\
\hline $\mathrm{H}$ & -8.00412 & 1.571561 & -1.29586 \\
\hline $\mathrm{H}$ & 11.01415 & 0.178799 & 0 \\
\hline $\mathrm{H}$ & 10.22405 & 1.471291 & -0.88787 \\
\hline $\mathrm{H}$ & 10.22405 & 1.471291 & 0.887867 \\
\hline $\mathrm{H}$ & 9.763006 & -1.51932 & 1.275875 \\
\hline $\mathrm{H}$ & 8.004119 & -1.57156 & 1.295861 \\
\hline $\mathrm{H}$ & 8.860452 & -0.29753 & 2.174754 \\
\hline $\mathrm{H}$ & 8.860452 & -0.29753 & -2.17475 \\
\hline $\mathrm{H}$ & 9.763006 & -1.51932 & -1.27588 \\
\hline $\mathrm{H}$ & 8.004119 & -1.57156 & -1.29586 \\
\hline
\end{tabular}


Table S9. Summary of the computational results.

\begin{tabular}{lll}
\hline Compound & $\begin{array}{l}\text { The number of } \\
\text { imaginary frequencies }\end{array}$ & $\begin{array}{l}\text { Computed total energies of the } \\
\text { optimized structure (hartree) }\end{array}$ \\
\hline $\mathbf{1}^{\prime}$ & 0 & -1530.696070 \\
$\mathbf{2}^{\prime}$ & 0 & -1530.691027 \\
$\mathbf{3}^{\prime}$ & 0 & -2163.637269 \\
$\mathbf{4}^{\prime}$ & 0 & -2163.632843 \\
$\mathbf{5}^{\prime}$ & 0 & -2675.280276 \\
$\mathbf{6}^{\prime}$ & 0 & -2828.992345 \\
\hline
\end{tabular}


NMR Spectra of All New Compounds

${ }^{1} \mathrm{H}$ NMR spectrum (400 MHz, CD 3 CN, $298 \mathrm{~K}$ )
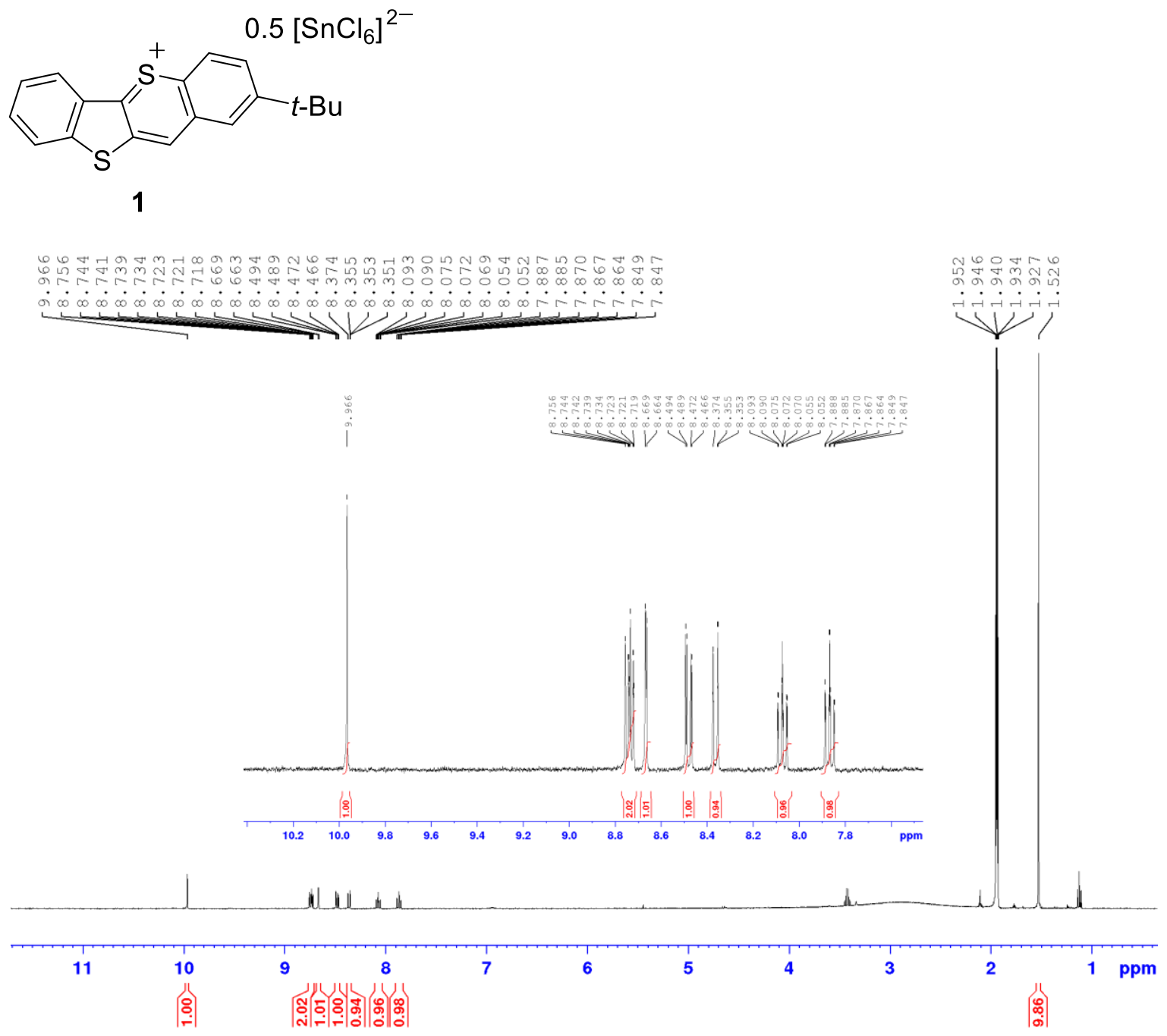
${ }^{13} \mathrm{C}\left\{{ }^{1} \mathrm{H}\right\}$ NMR spectrum (101 MHz, $\left.\mathrm{CD}_{3} \mathrm{CN}, 298 \mathrm{~K}\right)$
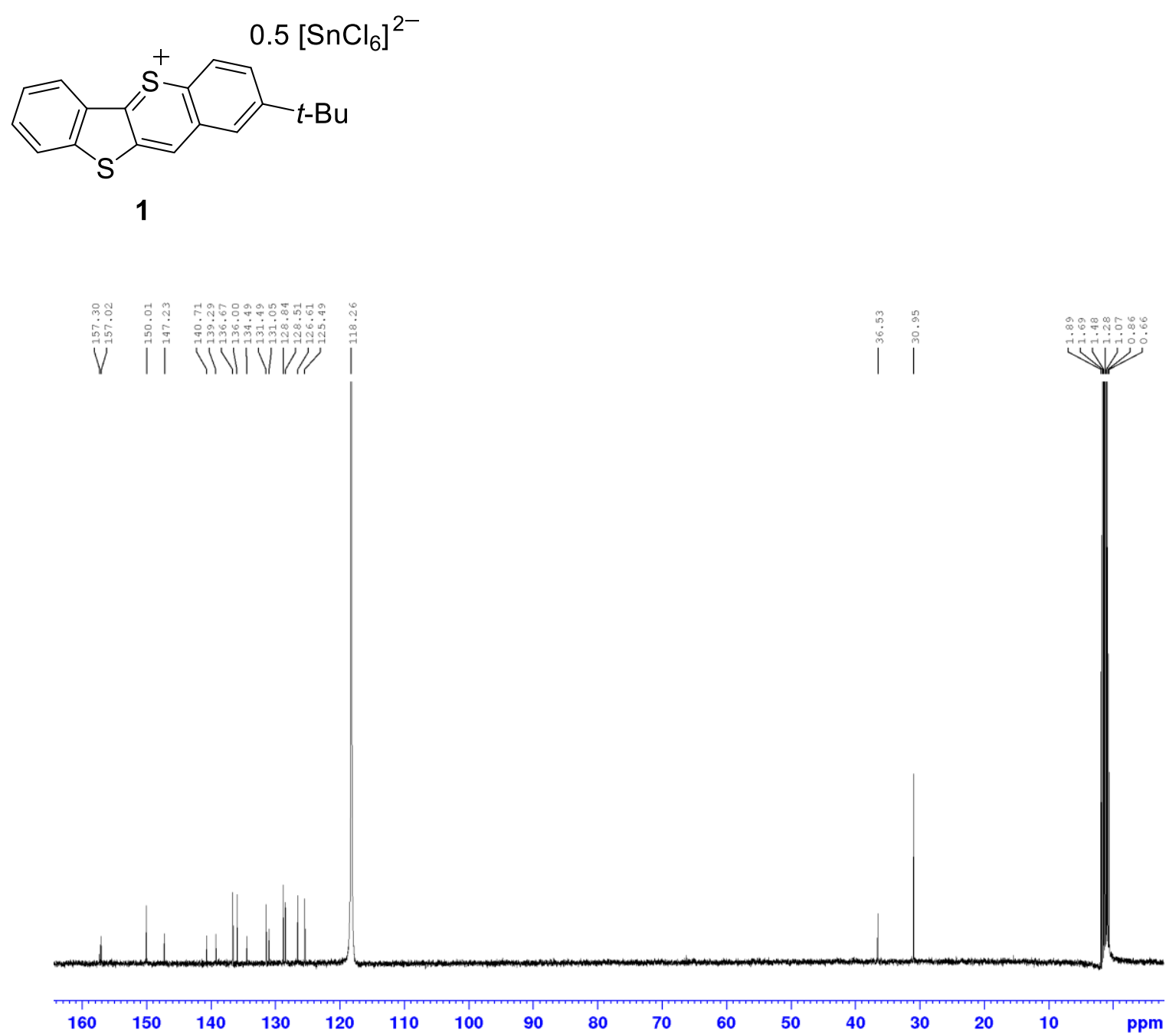
${ }^{1} \mathrm{H}$ NMR spectrum (400 MHz, CD 3 CN, $298 \mathrm{~K}$ )<smiles></smiles>
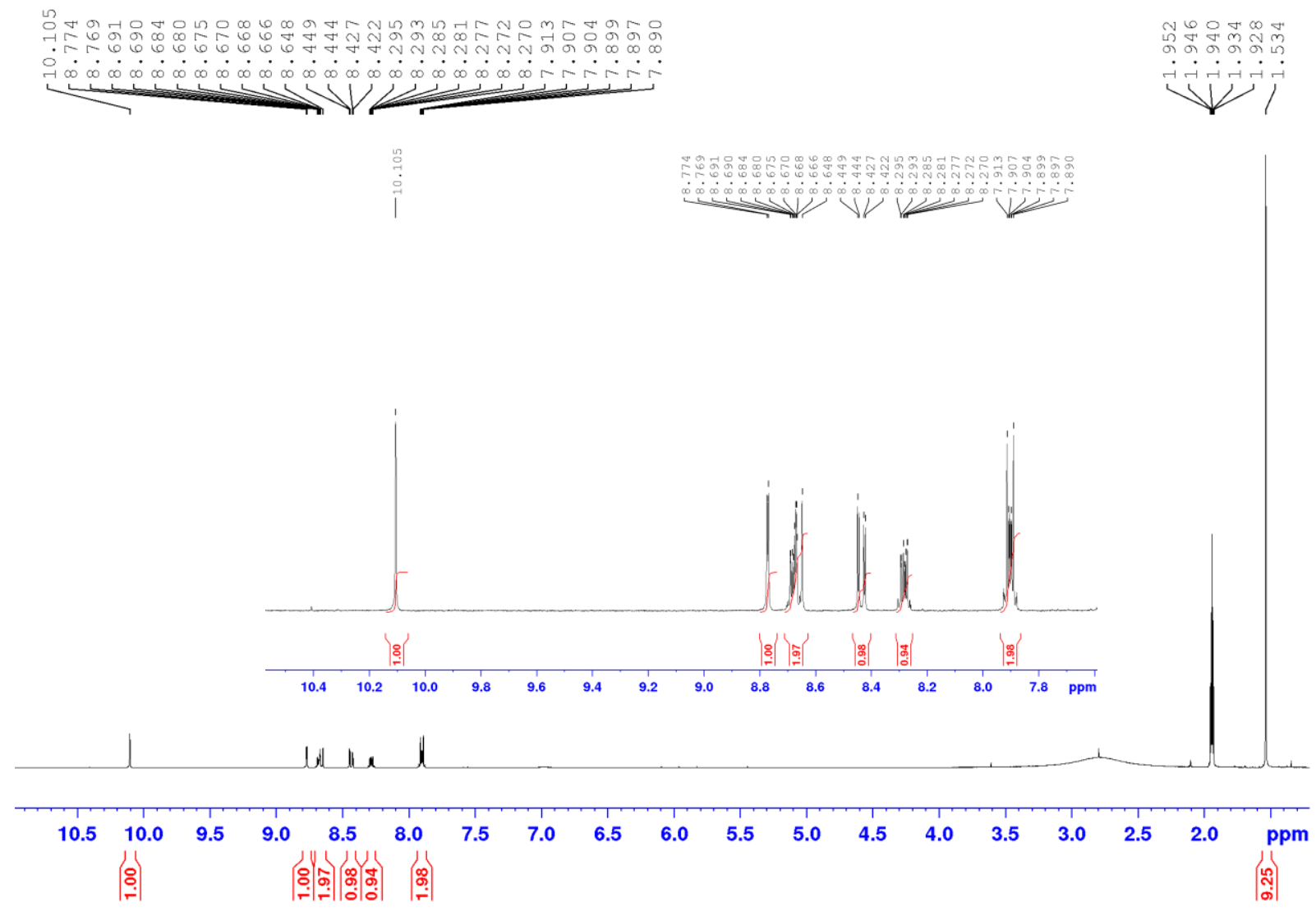
${ }^{13} \mathrm{C}\left\{{ }^{1} \mathrm{H}\right\}$ NMR spectrum (101 MHz, CD $3 \mathrm{CN}, 298 \mathrm{~K}$ )<smiles></smiles>

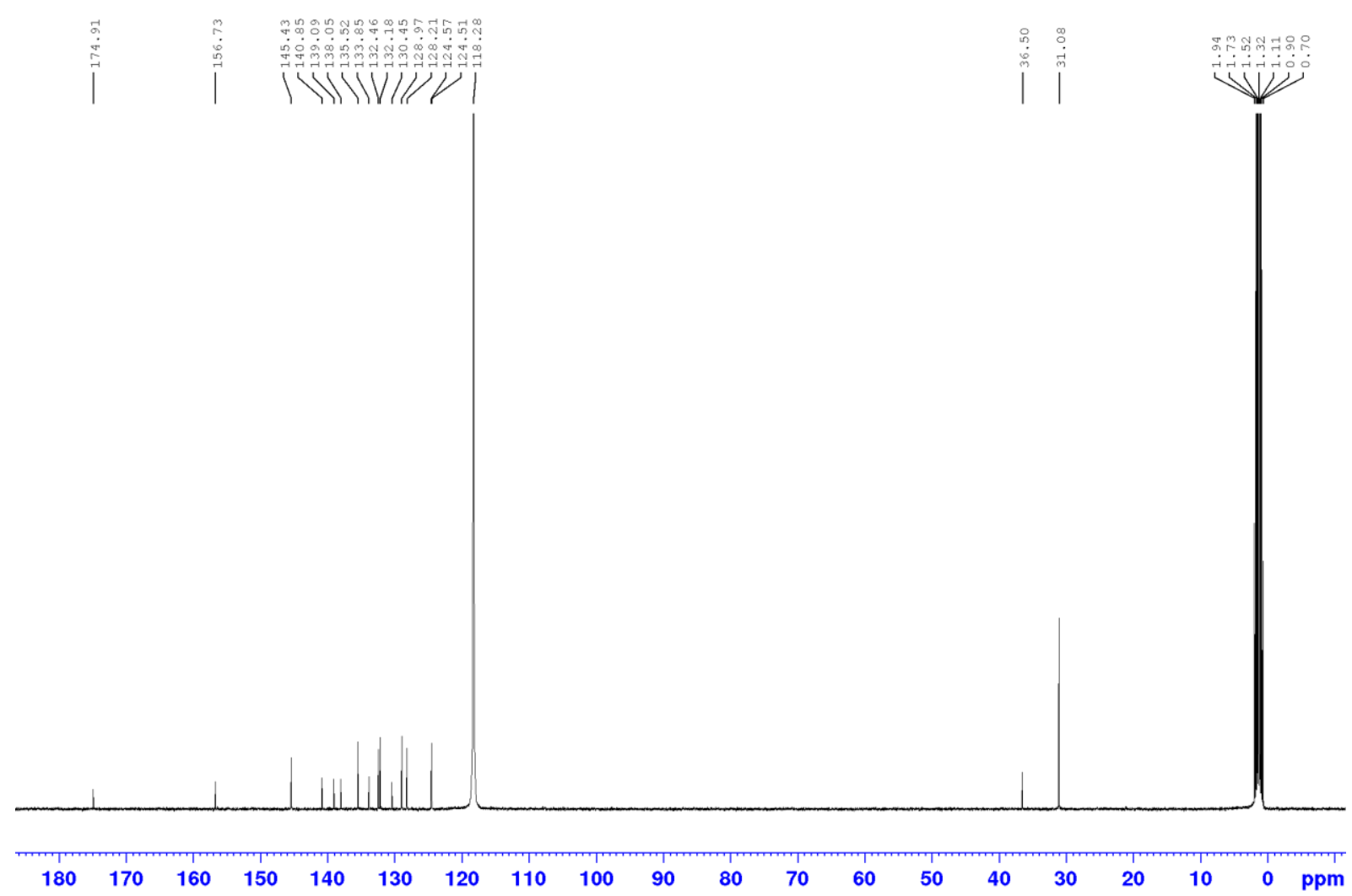


${ }^{1} \mathrm{H}$ NMR spectrum (400 MHz, CD 3 CN, $298 \mathrm{~K}$ )
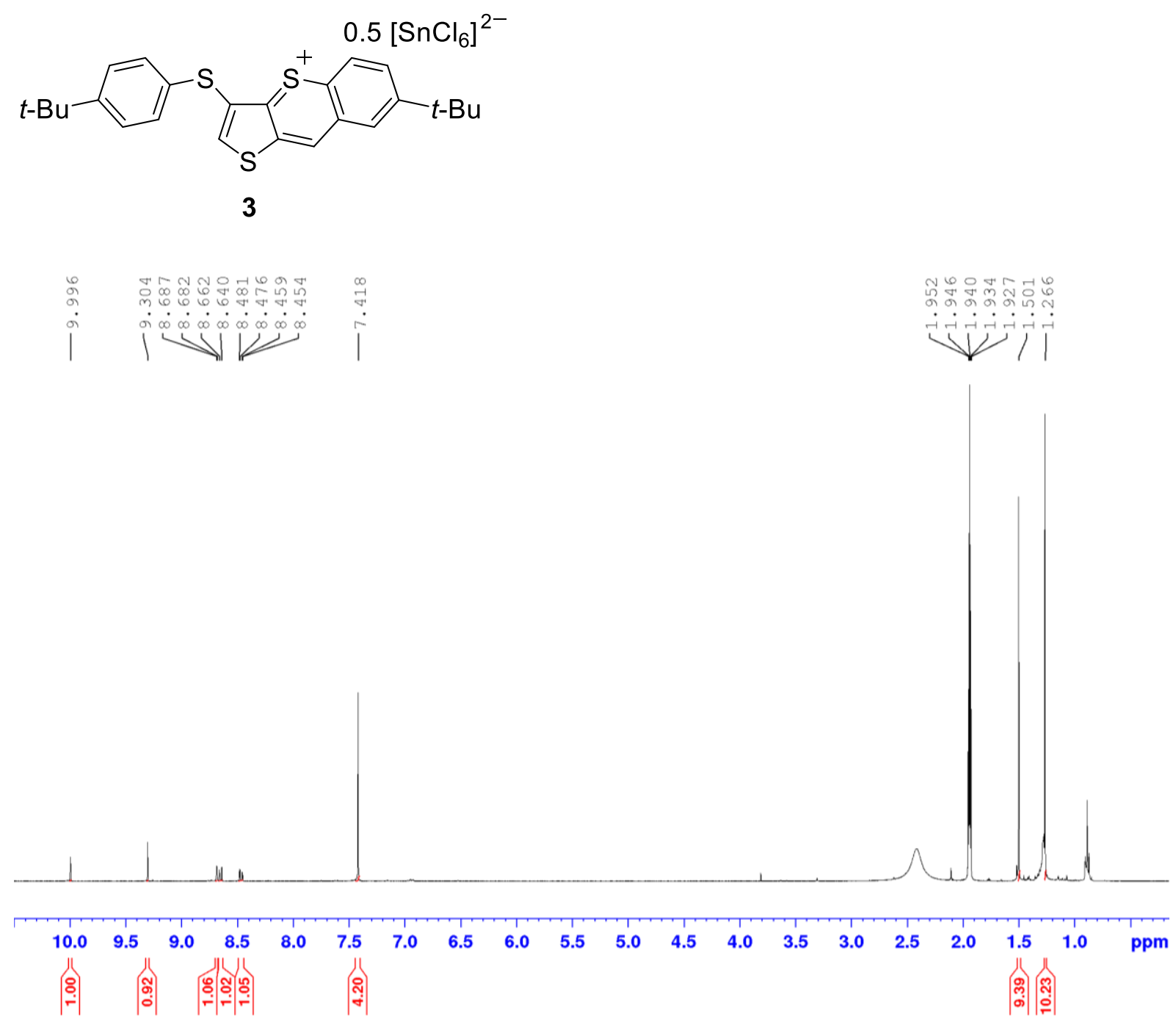
${ }^{13} \mathrm{C}\left\{{ }^{1} \mathrm{H}\right\}$ NMR spectrum (101 MHz, $\left.\mathrm{CD}_{3} \mathrm{CN}, 298 \mathrm{~K}\right)$<smiles>CC(C)(C)c1ccc(Sc2csc3[s+]c4ccc(C(C)(C)C)cc4[s+]c23)cc1</smiles>

|VII V|V VIV

川

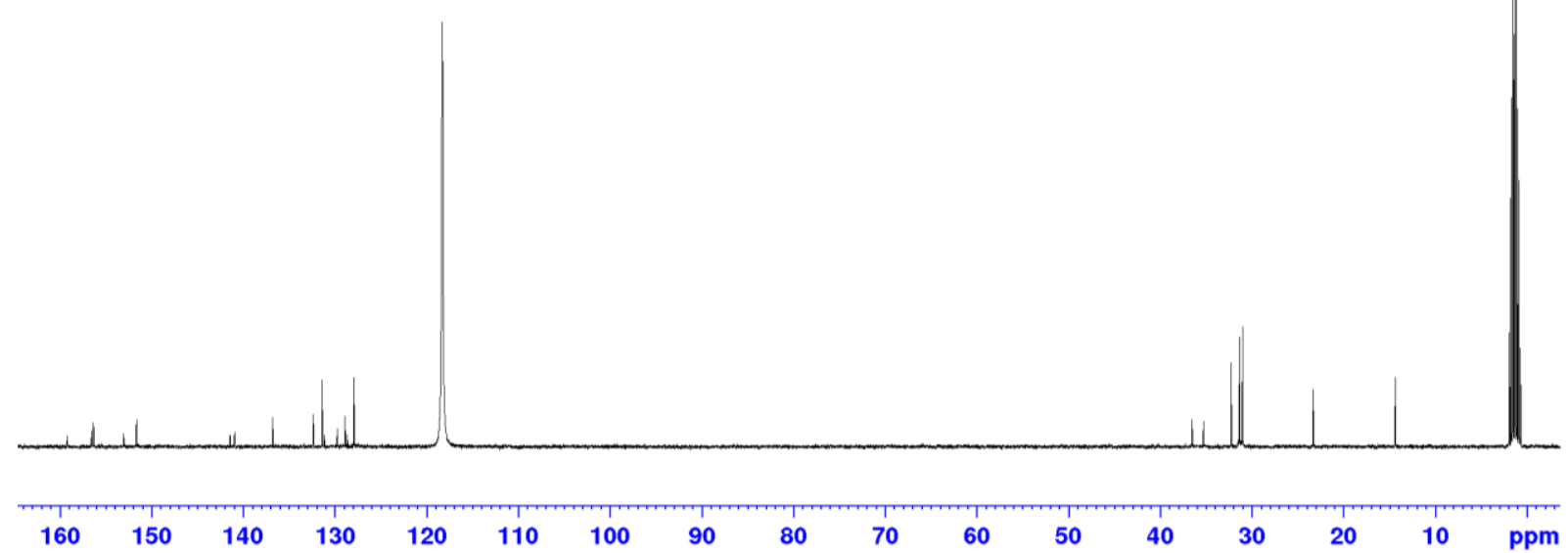


${ }^{1} \mathrm{H}$ NMR spectrum (400 MHz, CD 3 CN, $298 \mathrm{~K}$ )<smiles></smiles>

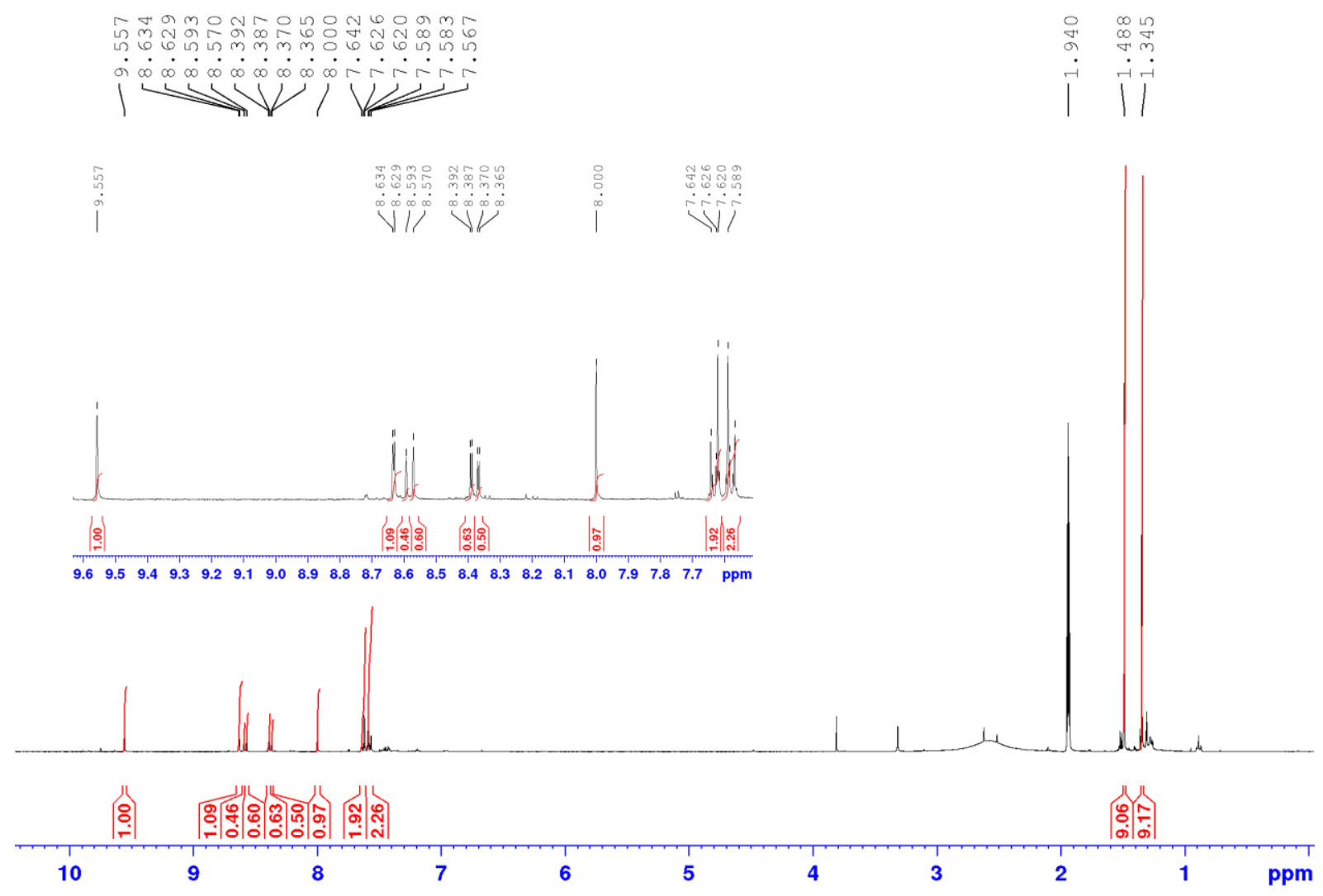


${ }^{13} \mathrm{C}\left\{{ }^{1} \mathrm{H}\right\}$ NMR spectrum (101 MHz, CD $3 \mathrm{CN}, 298 \mathrm{~K}$ )<smiles>CC(C)(C)c1ccc(Sc2cc3cc4cc(C(C)(C)C)ccc4[se]c-3c2[14CH2])cc1</smiles>

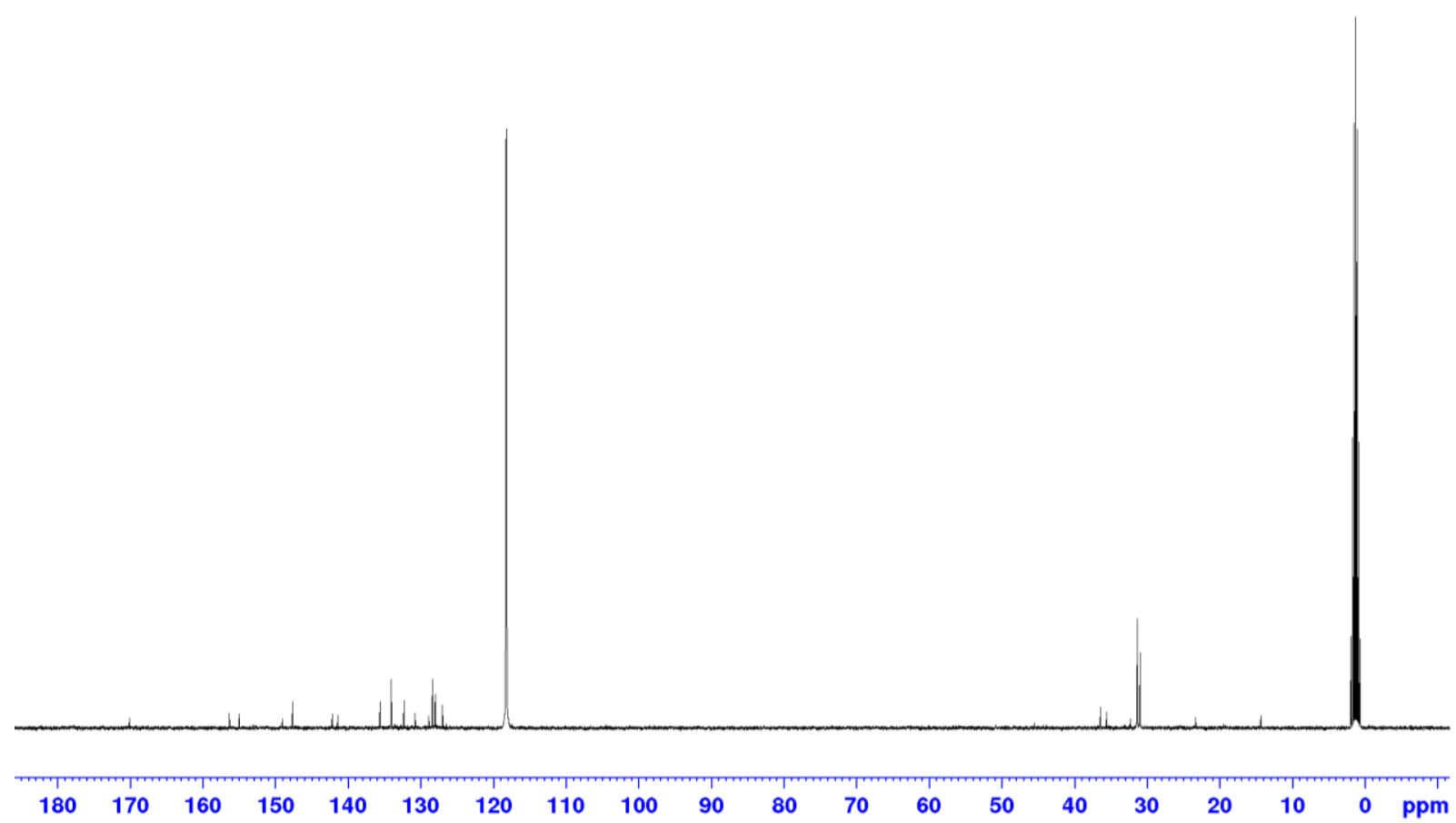


${ }^{1} \mathrm{H}$ NMR spectrum (400 MHz, CD 3 CN, $298 \mathrm{~K}$ )<smiles></smiles>

5
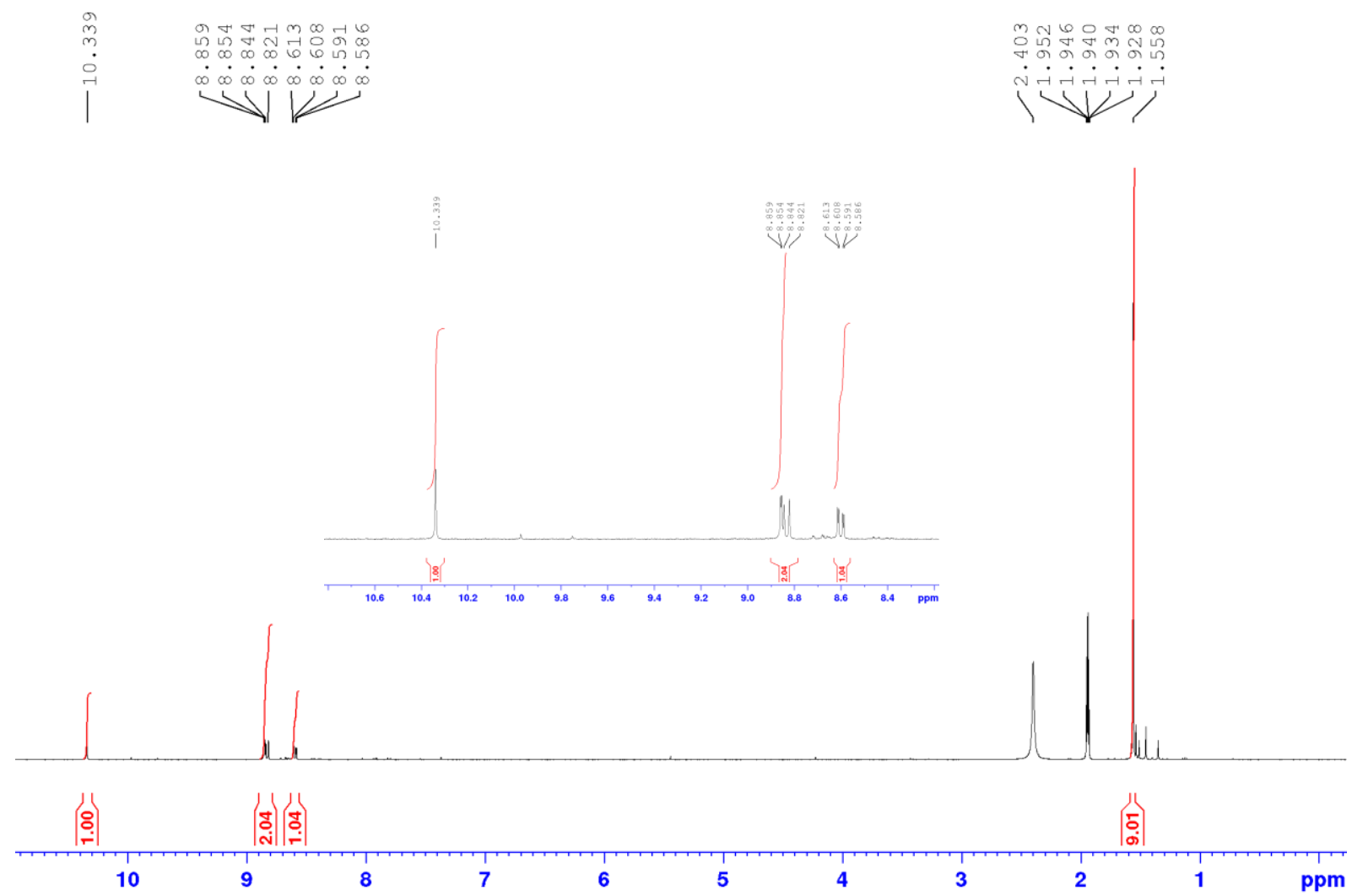
${ }^{1} \mathrm{H}$ NMR spectrum (400 MHz, CD 3 CN, $298 \mathrm{~K}$ )<smiles>CC(C)(C)c1ccc2[s+]c3sc4cc5c(cc4c3cc2c1)sc1[s+]c2ccc(C(C)(C)C)cc2cc15</smiles>

6
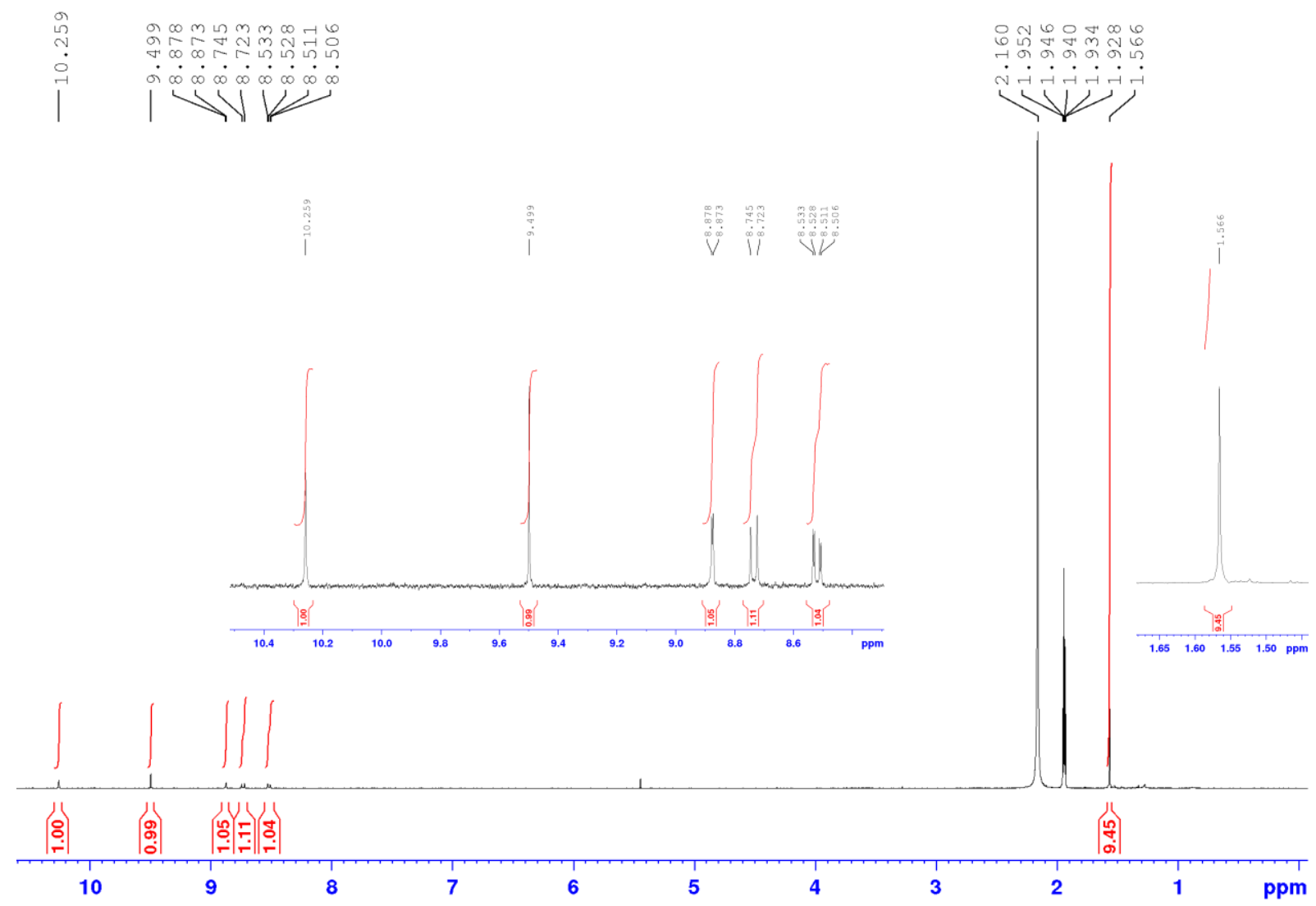
${ }^{1} \mathrm{H}$ NMR spectrum (400 MHz, $\mathrm{CDCl}_{3}, 298 \mathrm{~K}$ )
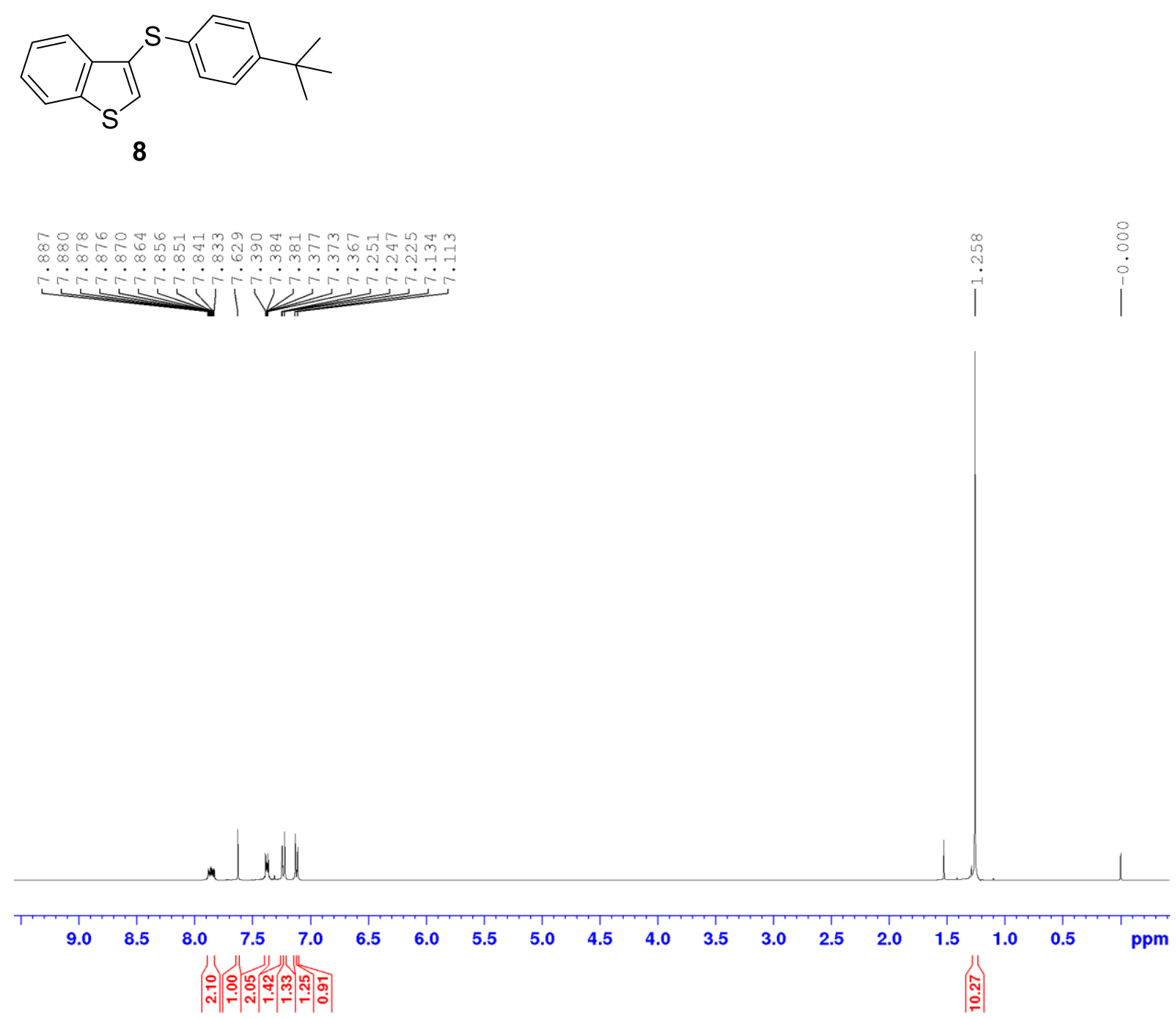
${ }^{13} \mathrm{C}\left\{{ }^{1} \mathrm{H}\right\}$ NMR spectrum $\left(101 \mathrm{MHz}, \mathrm{CDCl}_{3}, 298 \mathrm{~K}\right)$
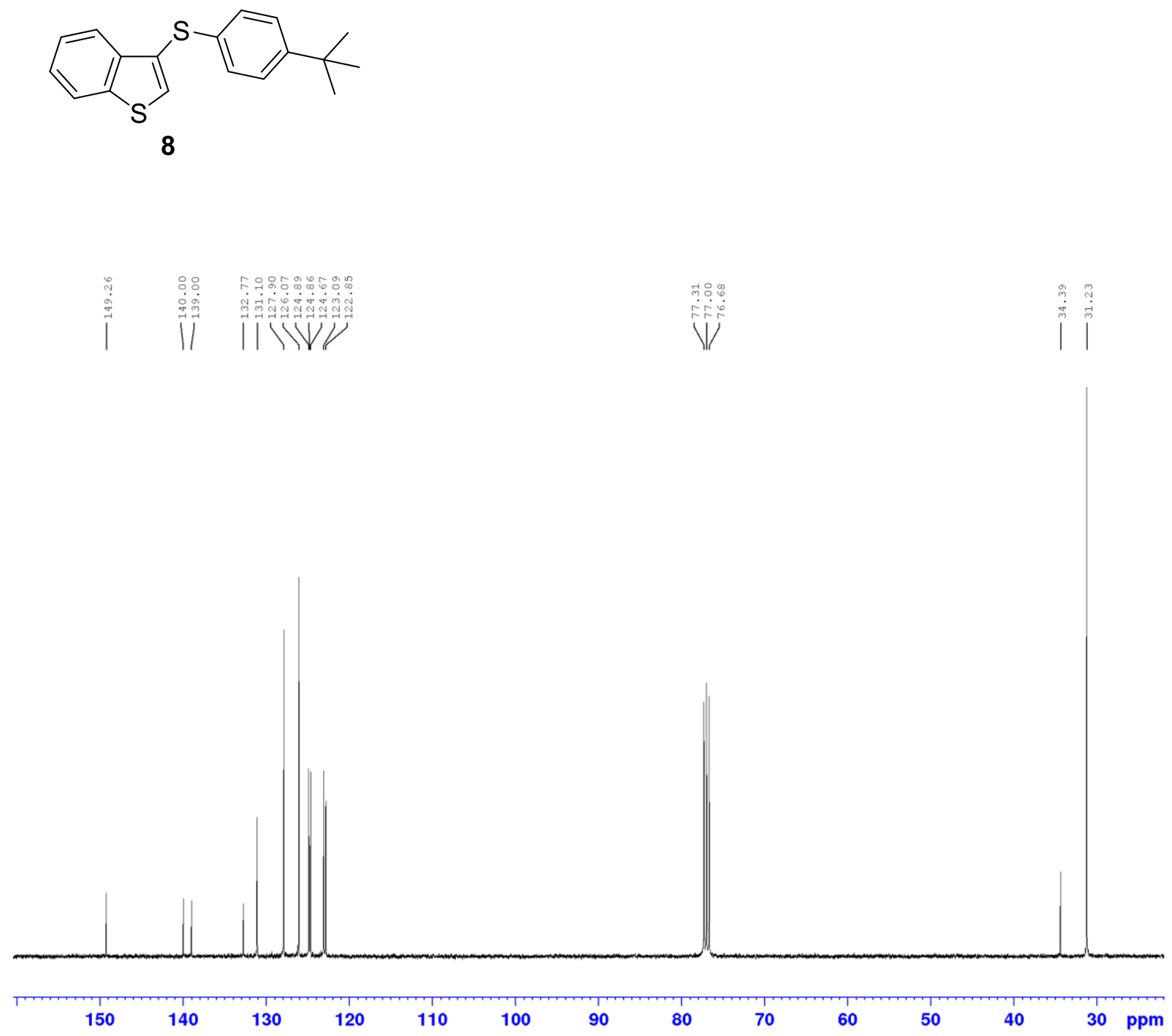
${ }^{1} \mathrm{H}$ NMR spectrum (400 MHz, $\mathrm{CDCl}_{3}, 298 \mathrm{~K}$ )
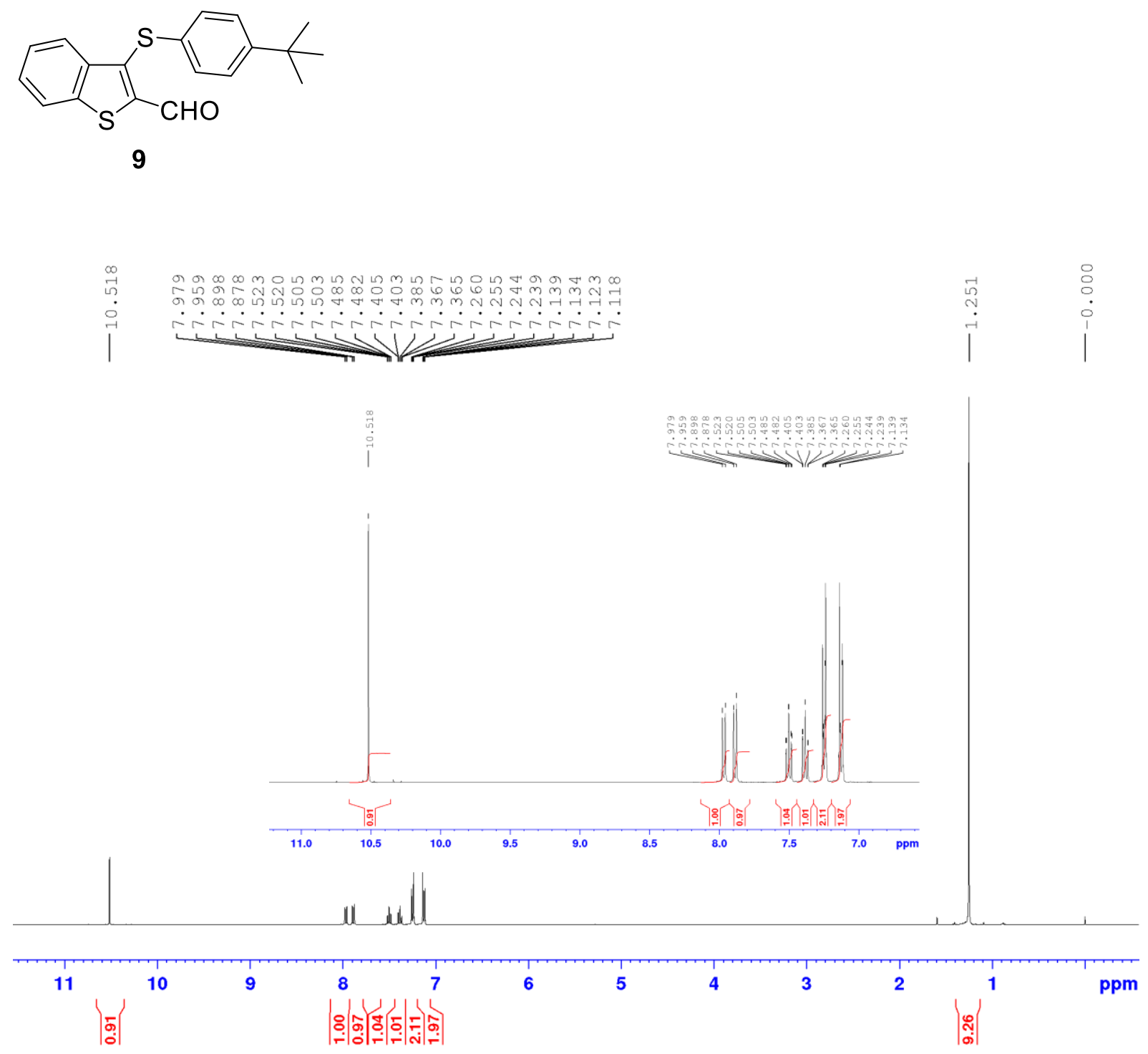
${ }^{13} \mathrm{C}\left\{{ }^{1} \mathrm{H}\right\}$ NMR spectrum (101 $\left.\mathrm{MHz}, \mathrm{CDCl}_{3}, 298 \mathrm{~K}\right)$
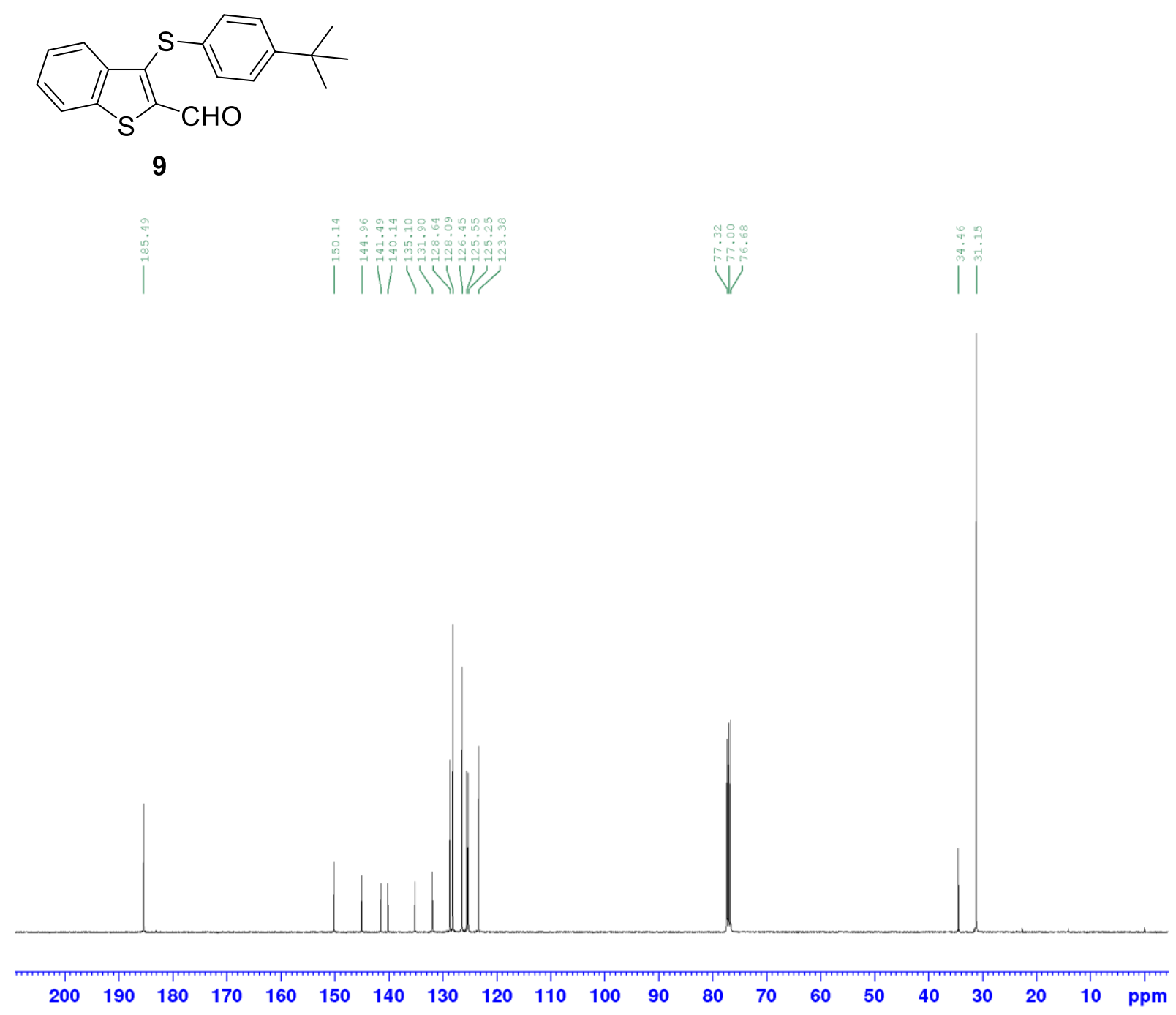
${ }^{1} \mathrm{H}$ NMR spectrum (400 MHz, $\mathrm{CDCl}_{3}, 298 \mathrm{~K}$ )

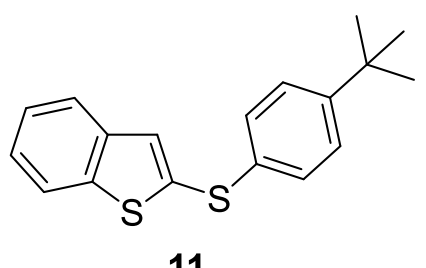

11

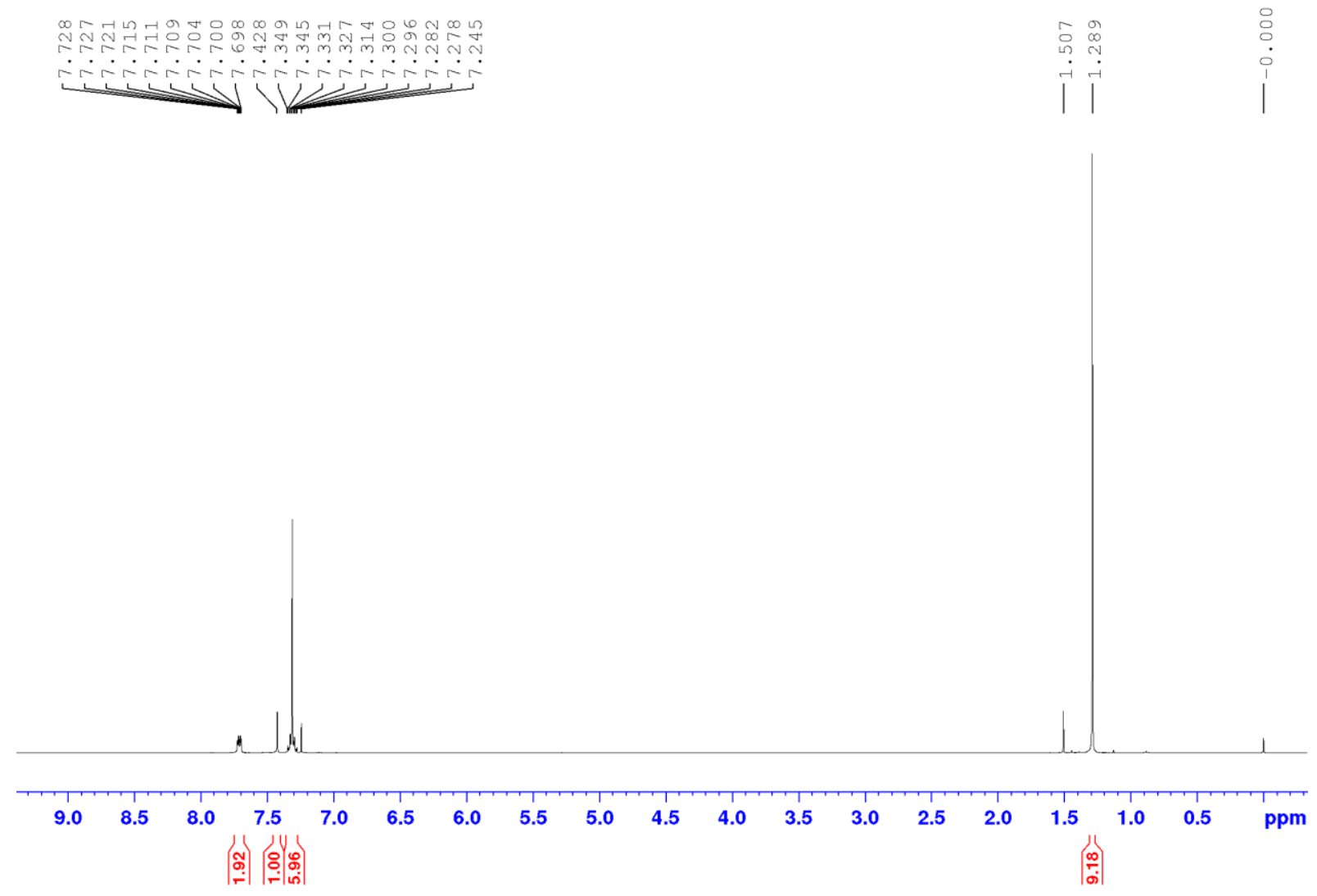


${ }^{13} \mathrm{C}\left\{{ }^{1} \mathrm{H}\right\} \mathrm{NMR}$ spectrum (101 MHz, $\left.\mathrm{CDCl}_{3}, 298 \mathrm{~K}\right)$<smiles>CC(C)(C)c1ccc(Sc2cc3ccccc3s2)cc1</smiles>

11

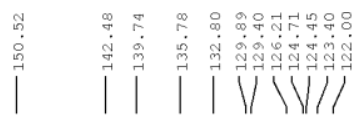

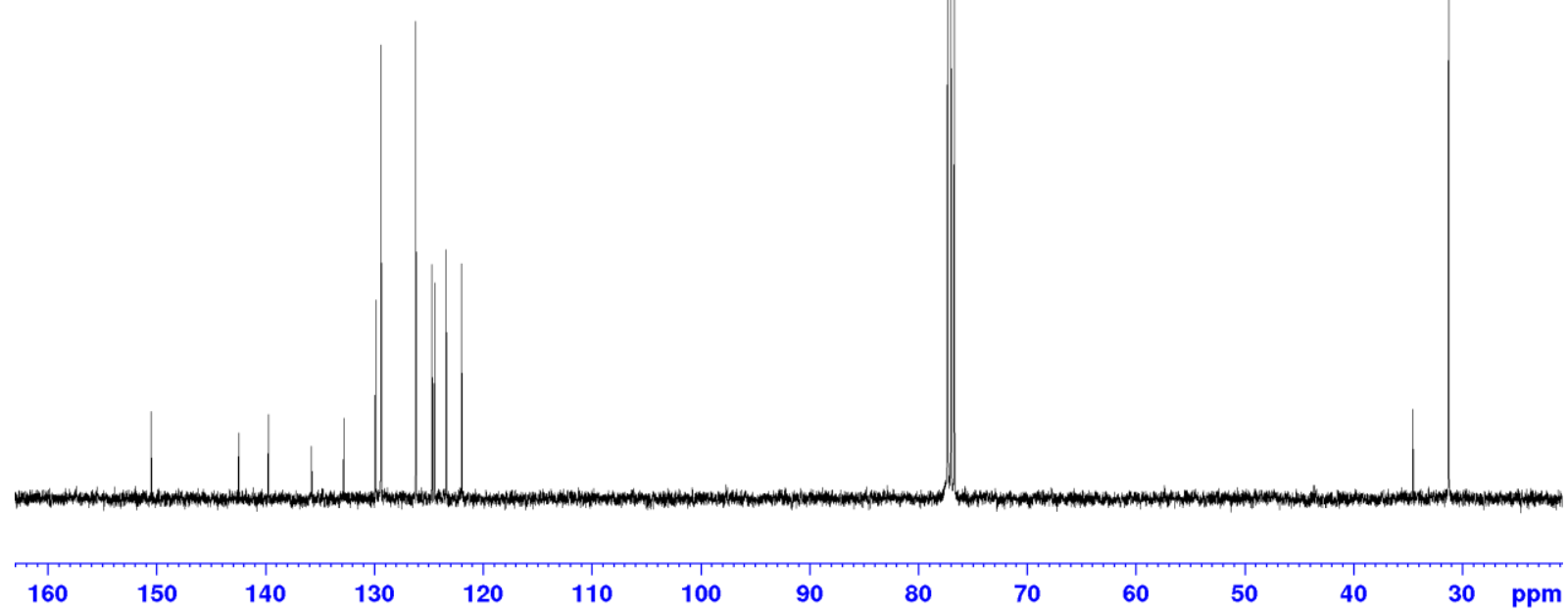


${ }^{1} \mathrm{H}$ NMR spectrum (400 MHz, $\mathrm{CDCl}_{3}, 298 \mathrm{~K}$ )
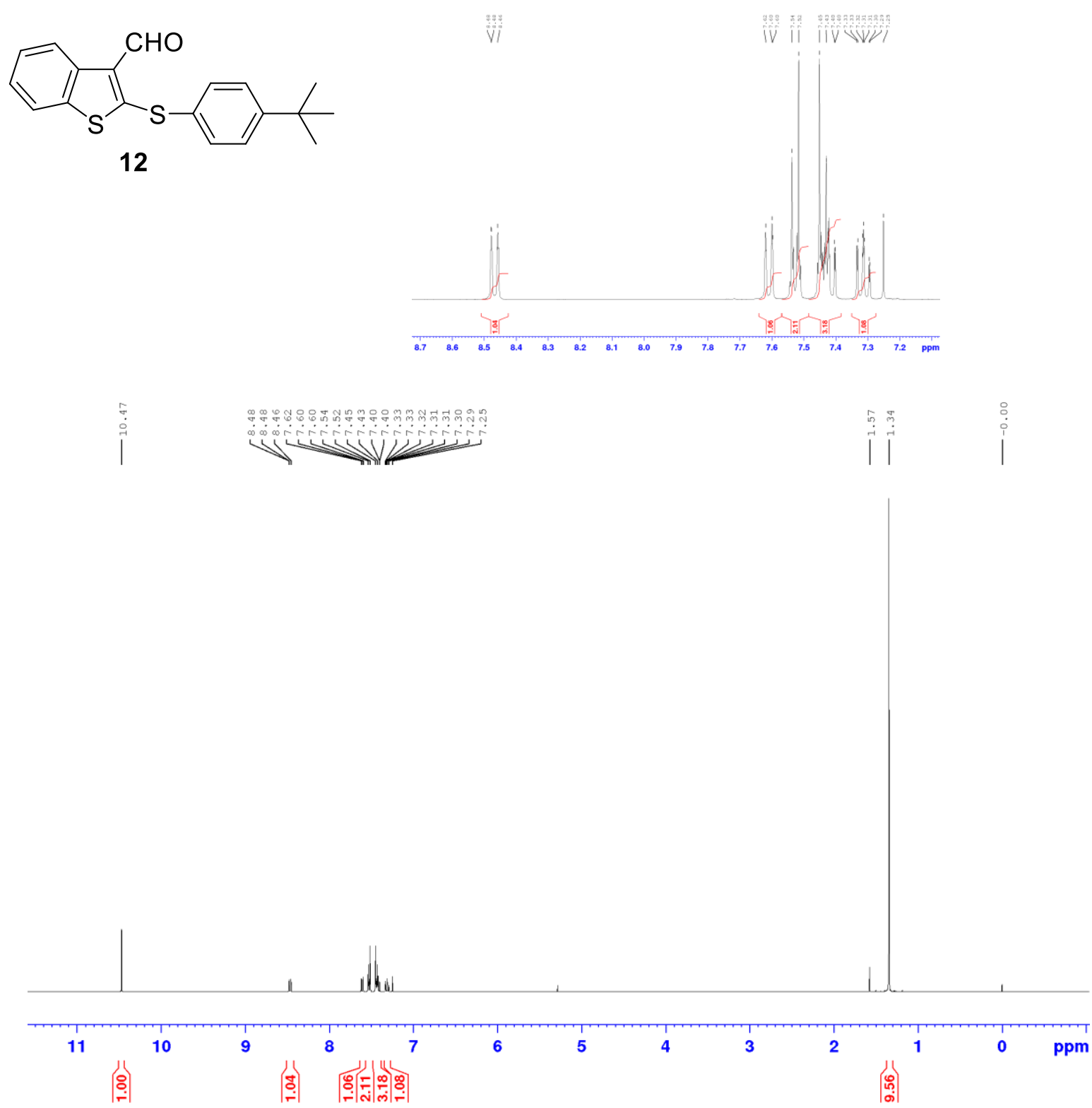
${ }^{13} \mathrm{C}\left\{{ }^{1} \mathrm{H}\right\} \mathrm{NMR}$ spectrum (101 $\left.\mathrm{MHz}, \mathrm{CDCl}_{3}, 298 \mathrm{~K}\right)$

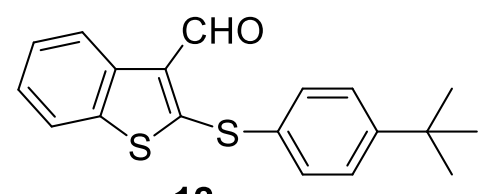

12

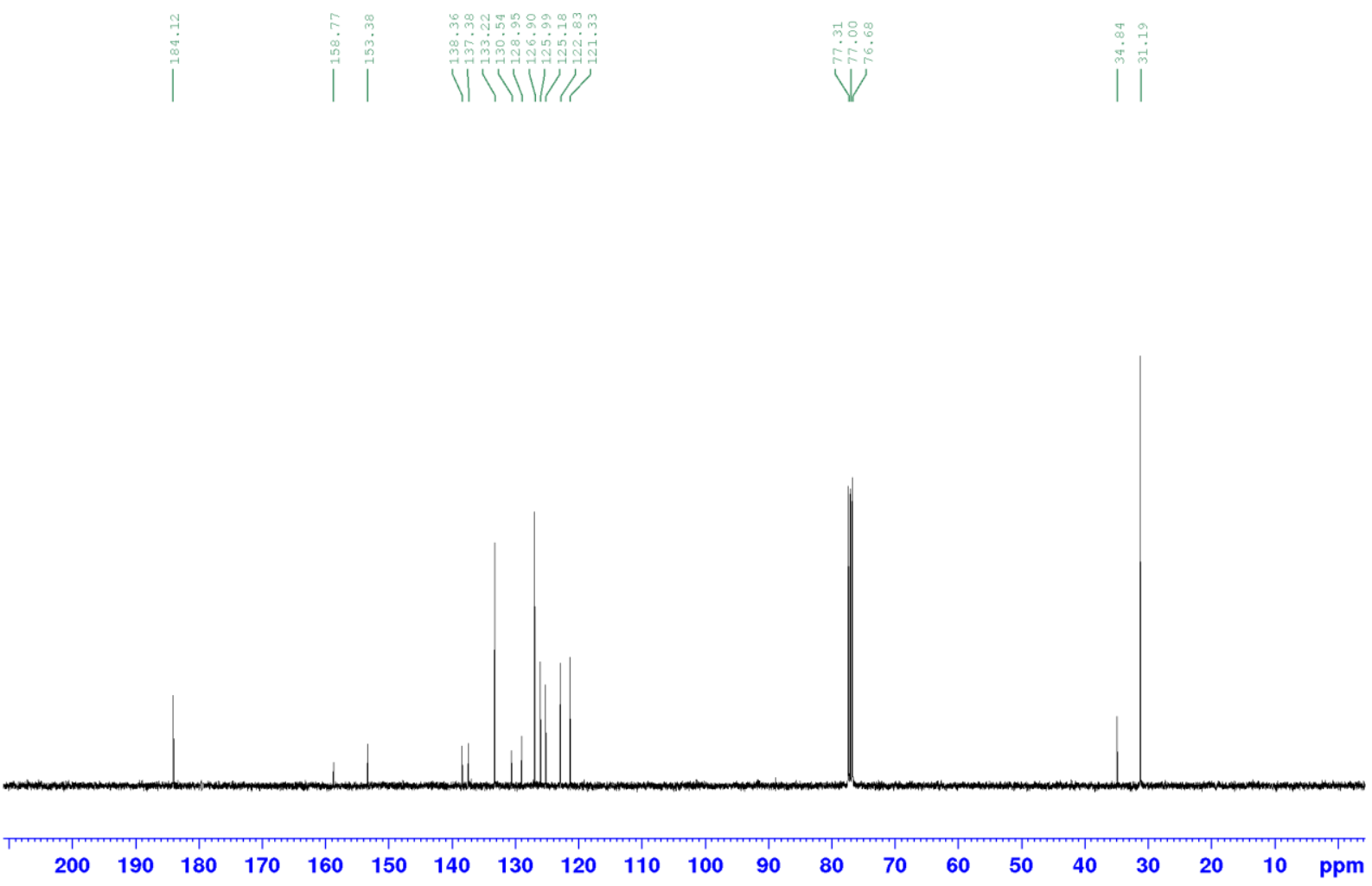


${ }^{1} \mathrm{H}$ NMR spectrum (400 MHz, $\mathrm{CDCl}_{3}, 298 \mathrm{~K}$ )

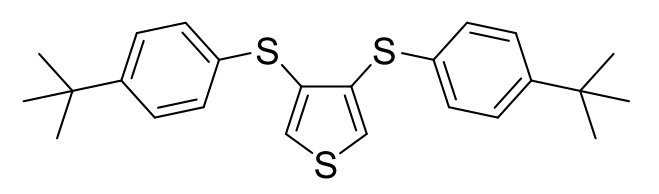

17

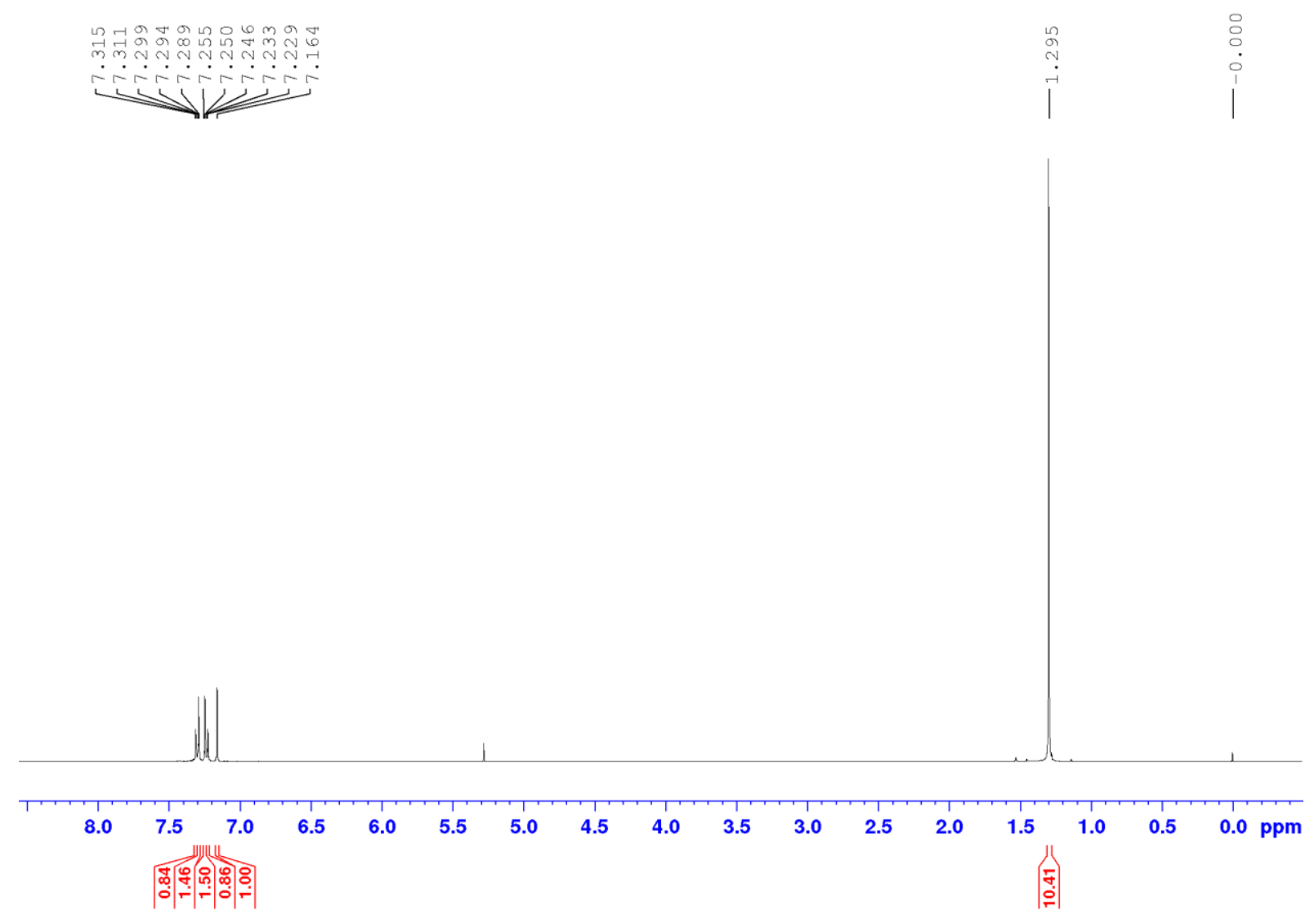


${ }^{13} \mathrm{C}\left\{{ }^{1} \mathrm{H}\right\}$ NMR spectrum (101 $\left.\mathrm{MHz}, \mathrm{CDCl}_{3}, 298 \mathrm{~K}\right)$<smiles>CC(C)(C)c1ccc(Sc2cscc2Sc2ccc(C(C)(C)C)cc2)cc1</smiles>

17

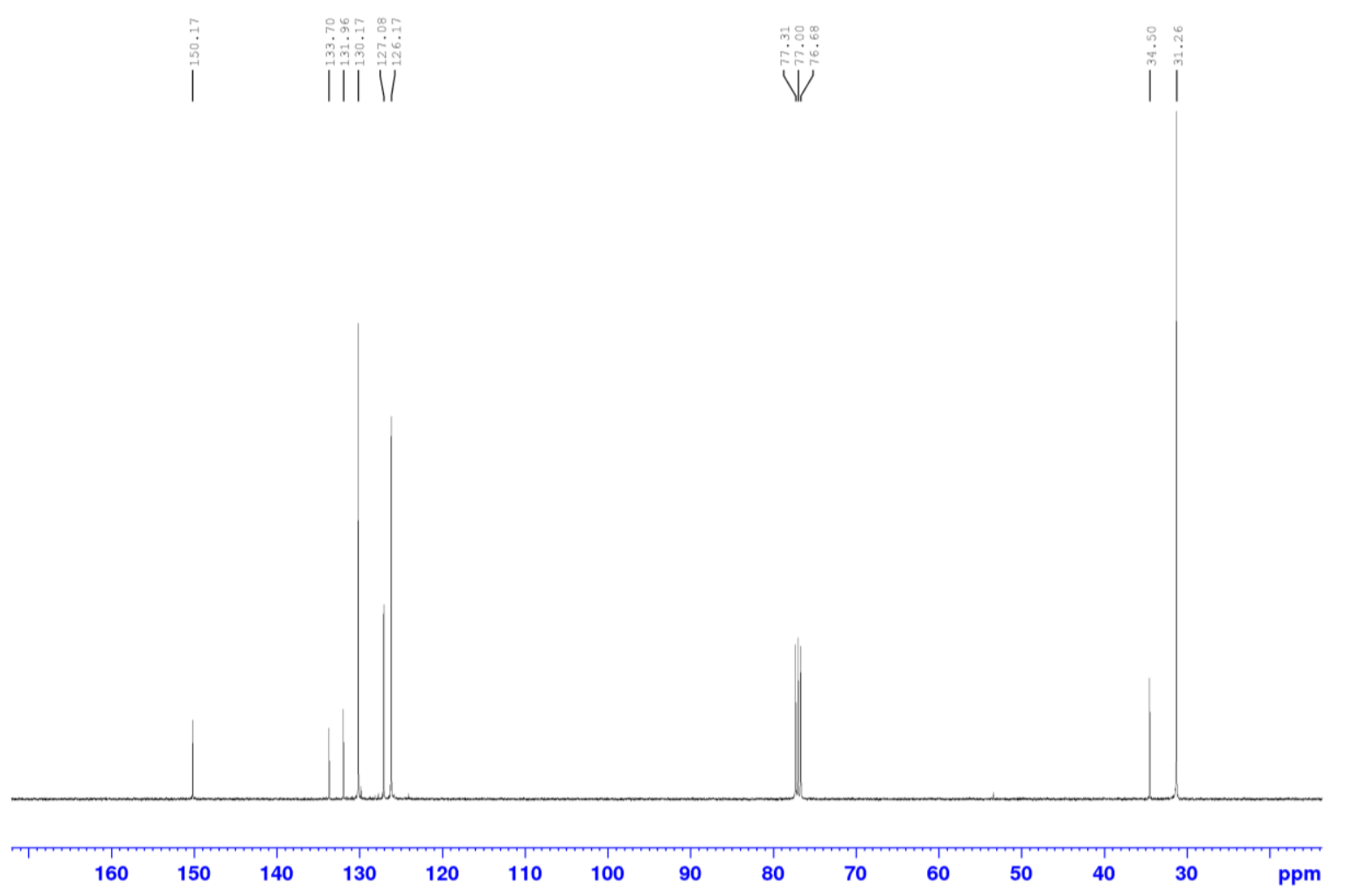


${ }^{1} \mathrm{H}$ NMR spectrum (400 MHz, $\mathrm{CDCl}_{3}, 298 \mathrm{~K}$ )<smiles>CC(C)(C)c1ccc(Sc2ccc(Sc3ccc(C(C)(C)C)cc3)s2)cc1</smiles>

18

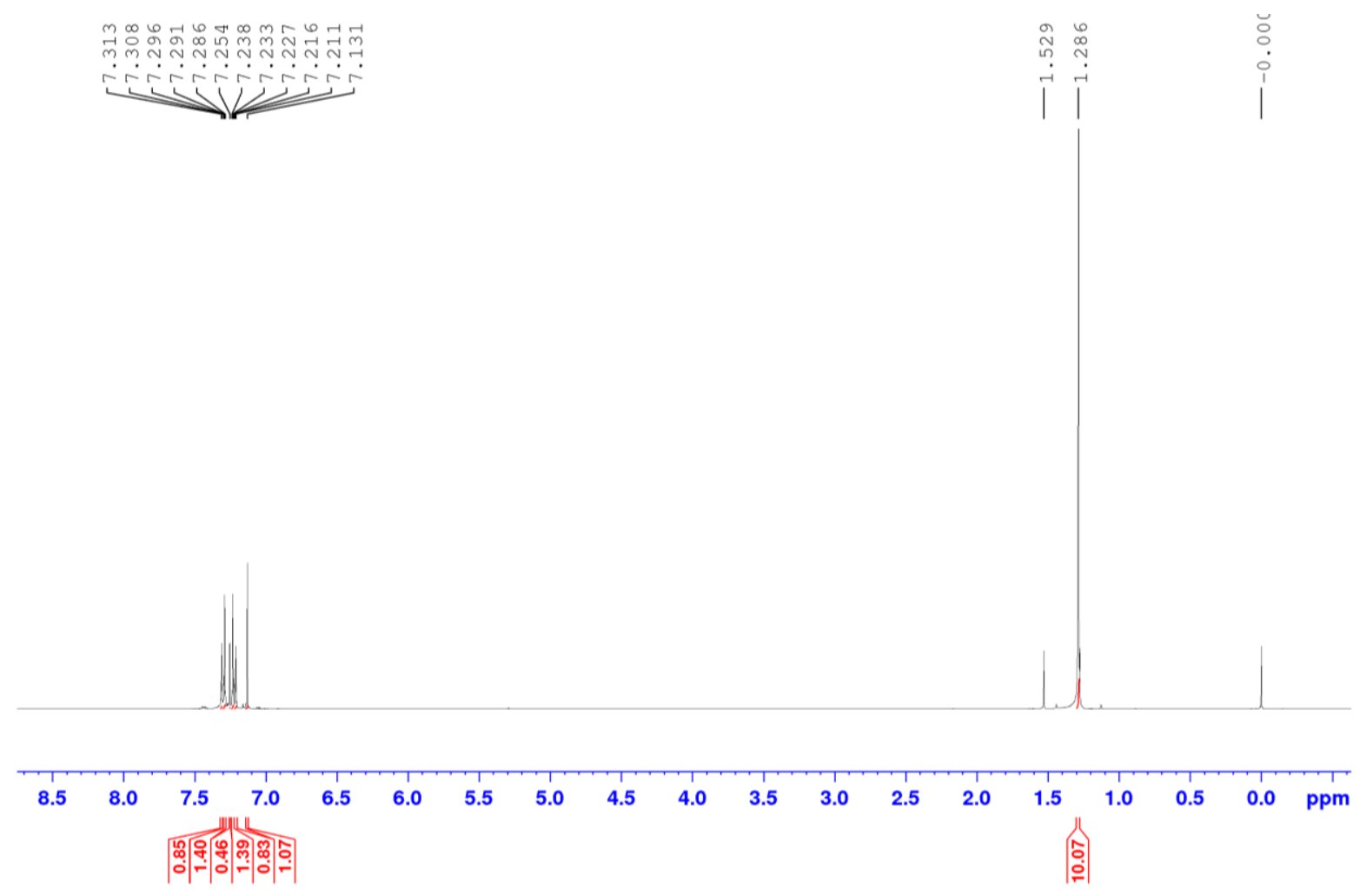


${ }^{13} \mathrm{C}\left\{{ }^{1} \mathrm{H}\right\}$ NMR spectrum (101 MHz, $\left.\mathrm{CDCl}_{3}, 298 \mathrm{~K}\right)$

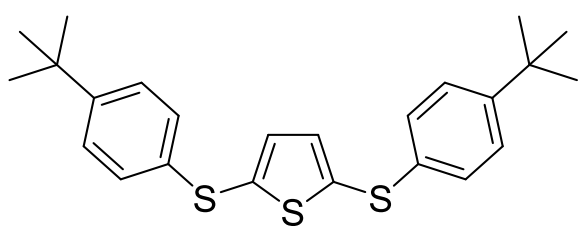

18

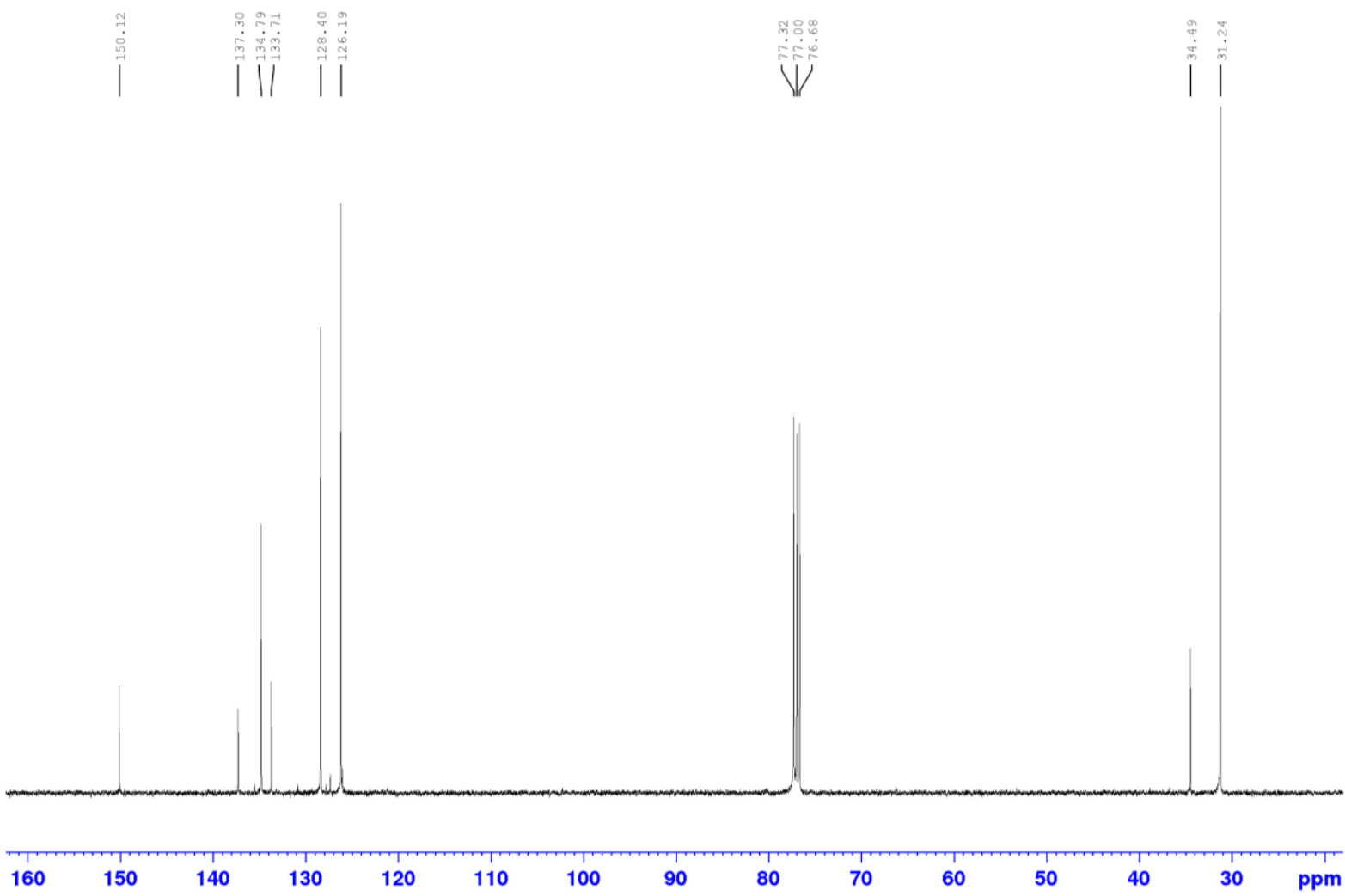


${ }^{1} \mathrm{H}$ NMR spectrum (400 MHz, $\mathrm{CDCl}_{3}, 298 \mathrm{~K}$ )
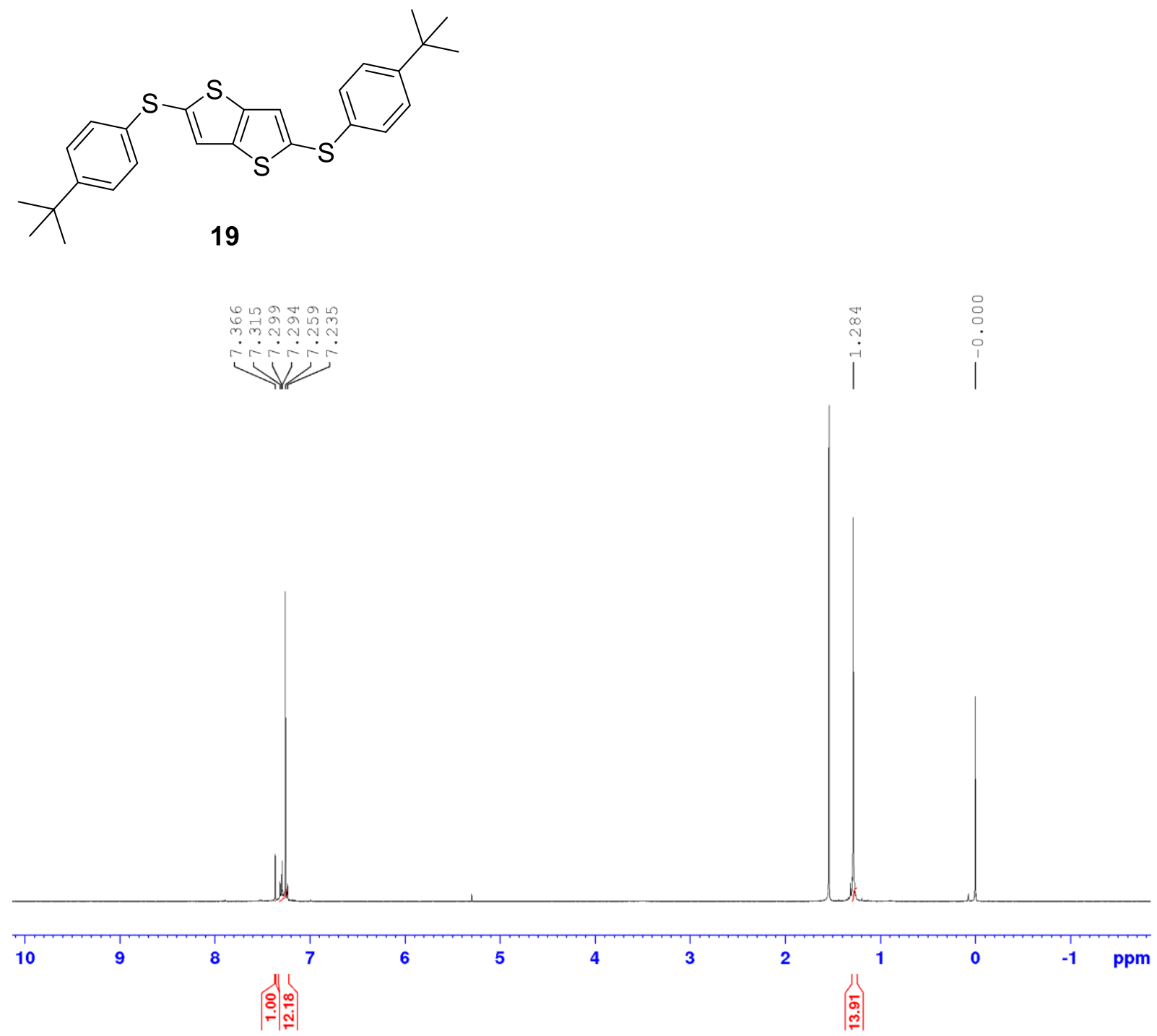
${ }^{13} \mathrm{C}\left\{{ }^{1} \mathrm{H}\right\} \mathrm{NMR}$ spectrum (101 MHz, $\left.\mathrm{CDCl}_{3}, 298 \mathrm{~K}\right)$<smiles>CC(C)(C)c1ccc(Sc2cc3sc(Sc4ccc(C(C)(C)C)cc4)cc3s2)cc1</smiles>

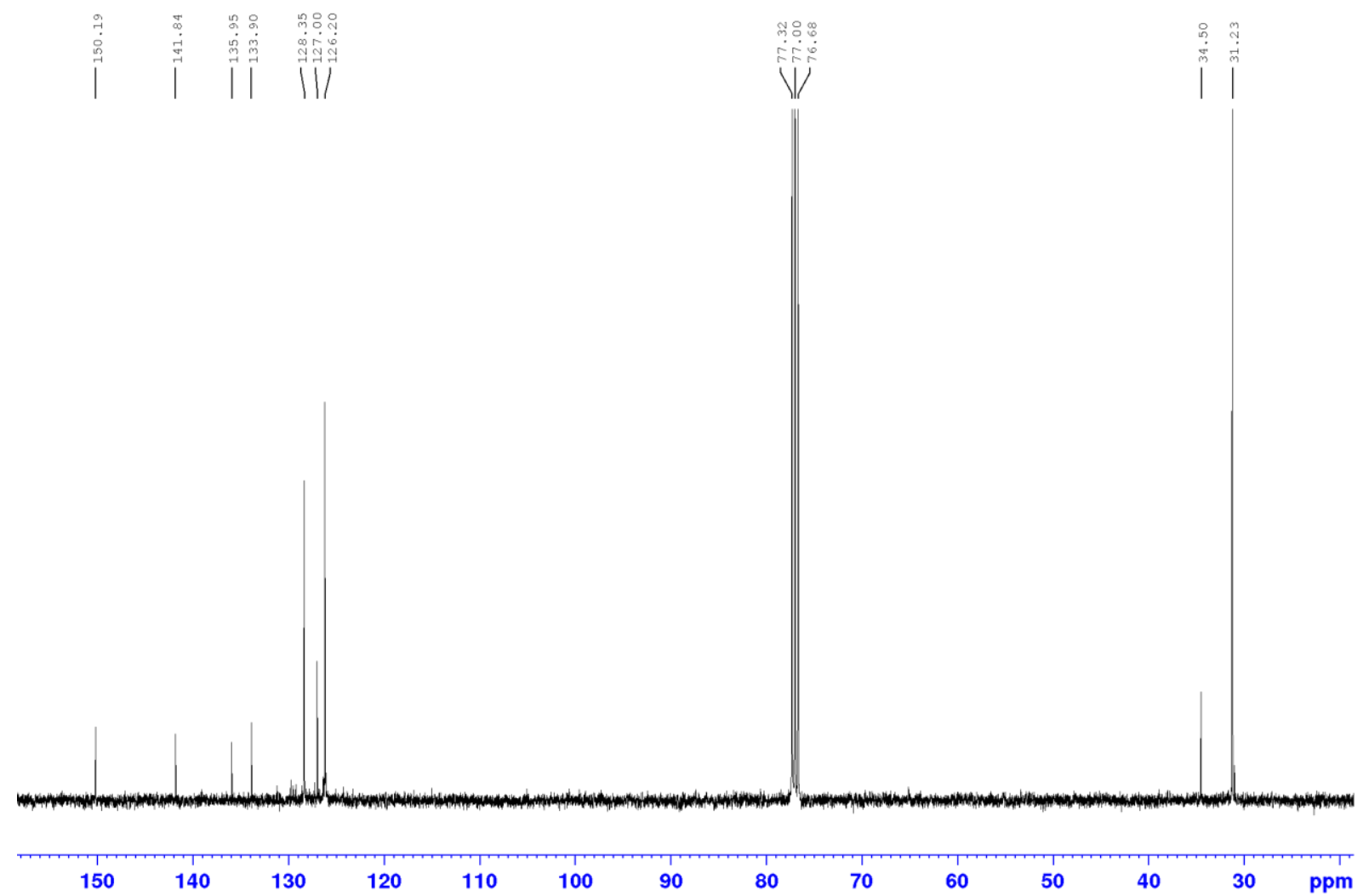


${ }^{1} \mathrm{H}$ NMR spectrum (400 MHz, $\mathrm{CDCl}_{3}, 298 \mathrm{~K}$ )

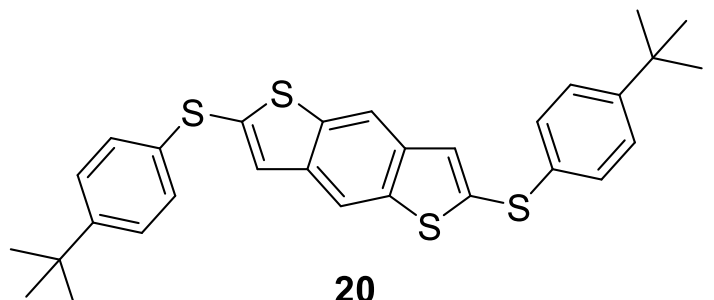

20

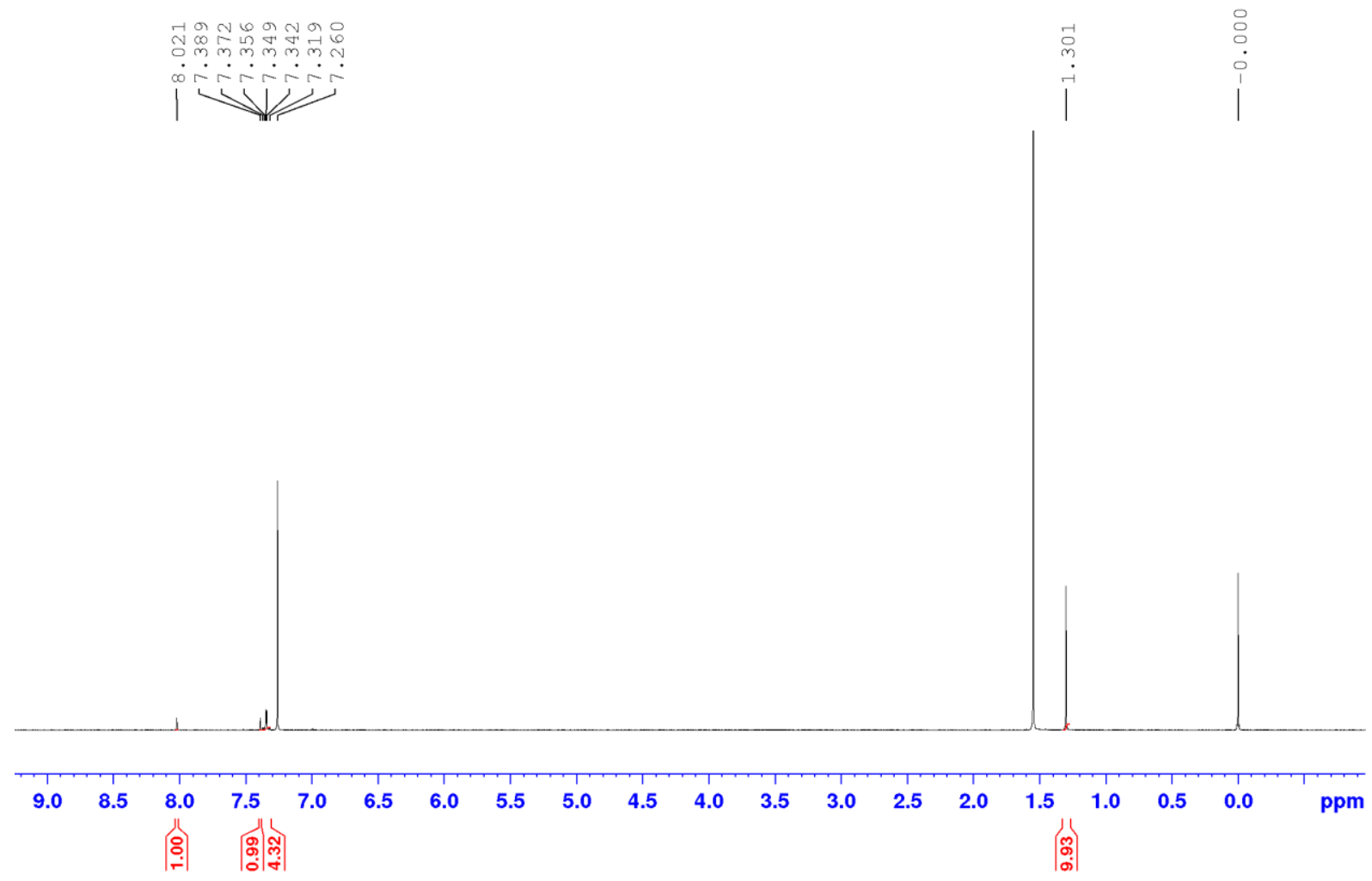


${ }^{13} \mathrm{C}\left\{{ }^{1} \mathrm{H}\right\} \mathrm{NMR}$ spectrum (101 MHz, $\left.\mathrm{CDCl}_{3}, 298 \mathrm{~K}\right)$<smiles>CC(C)(C)c1ccc(Sc2cc3cc4sc(Sc5ccc(C(C)(C)C)cc5)cc4cc3s2)cc1</smiles>

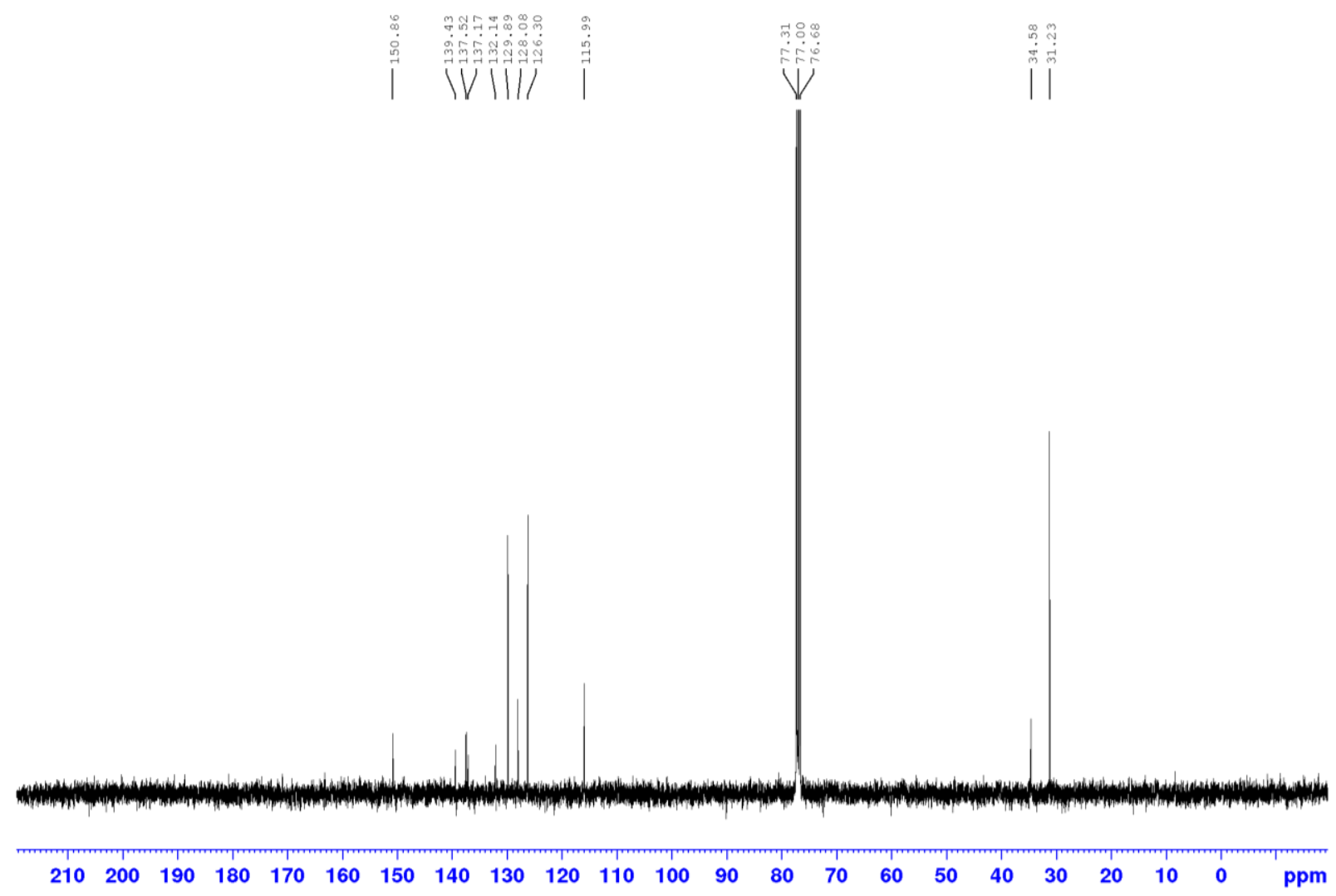


${ }^{1} \mathrm{H}$ NMR spectrum (400 MHz, $\mathrm{CDCl}_{3}, 298 \mathrm{~K}$ )<smiles>Cc1c(Sc2ccc(C(C)(C)C)cc2)sc2c(C)c(Sc3ccc(C(C)(C)C)cc3)sc12</smiles>

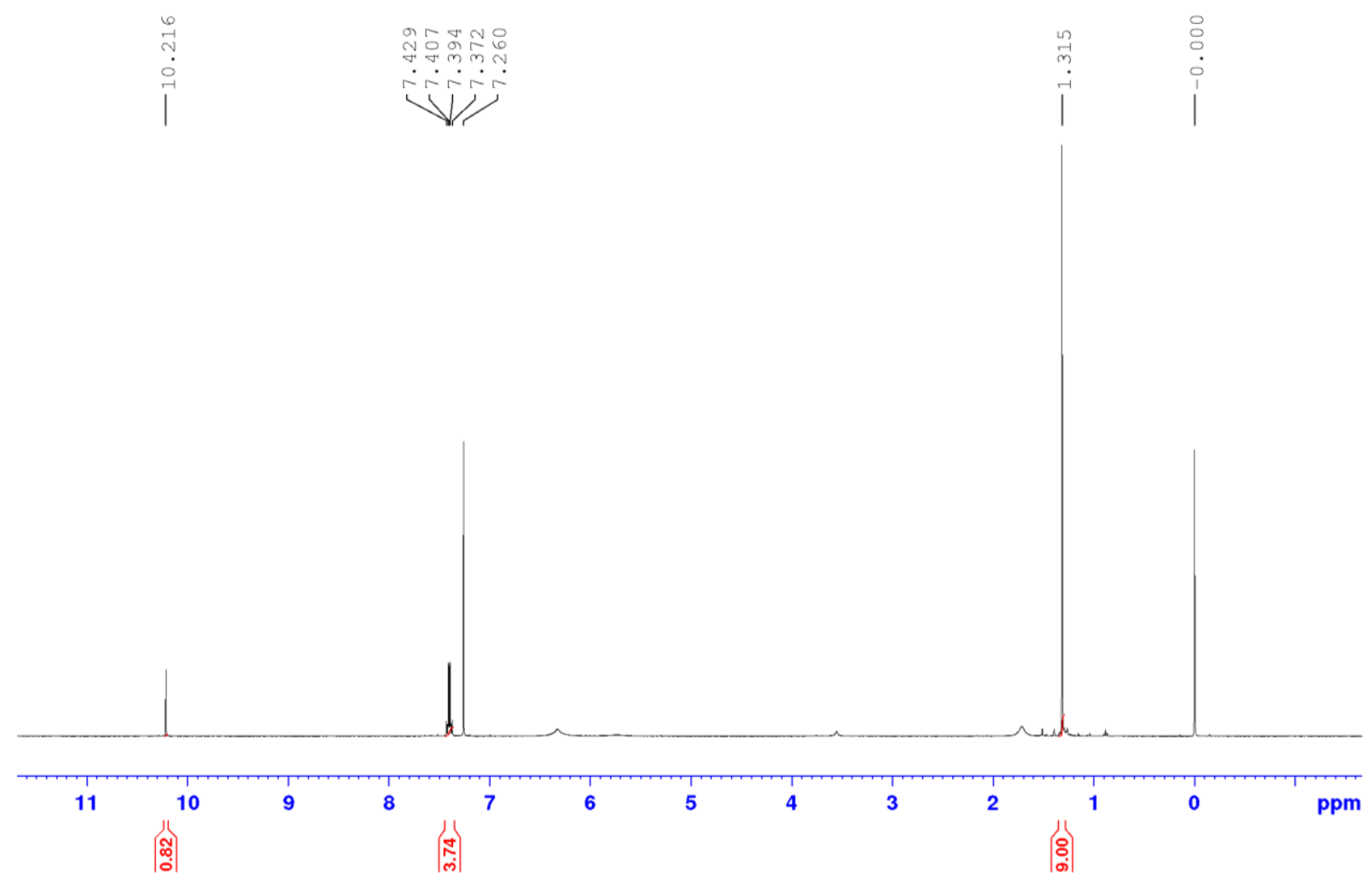


${ }^{13} \mathrm{C}\left\{{ }^{1} \mathrm{H}\right\} \mathrm{NMR}$ spectrum (101 MHz, $\left.\mathrm{CDCl}_{3}, 298 \mathrm{~K}\right)$
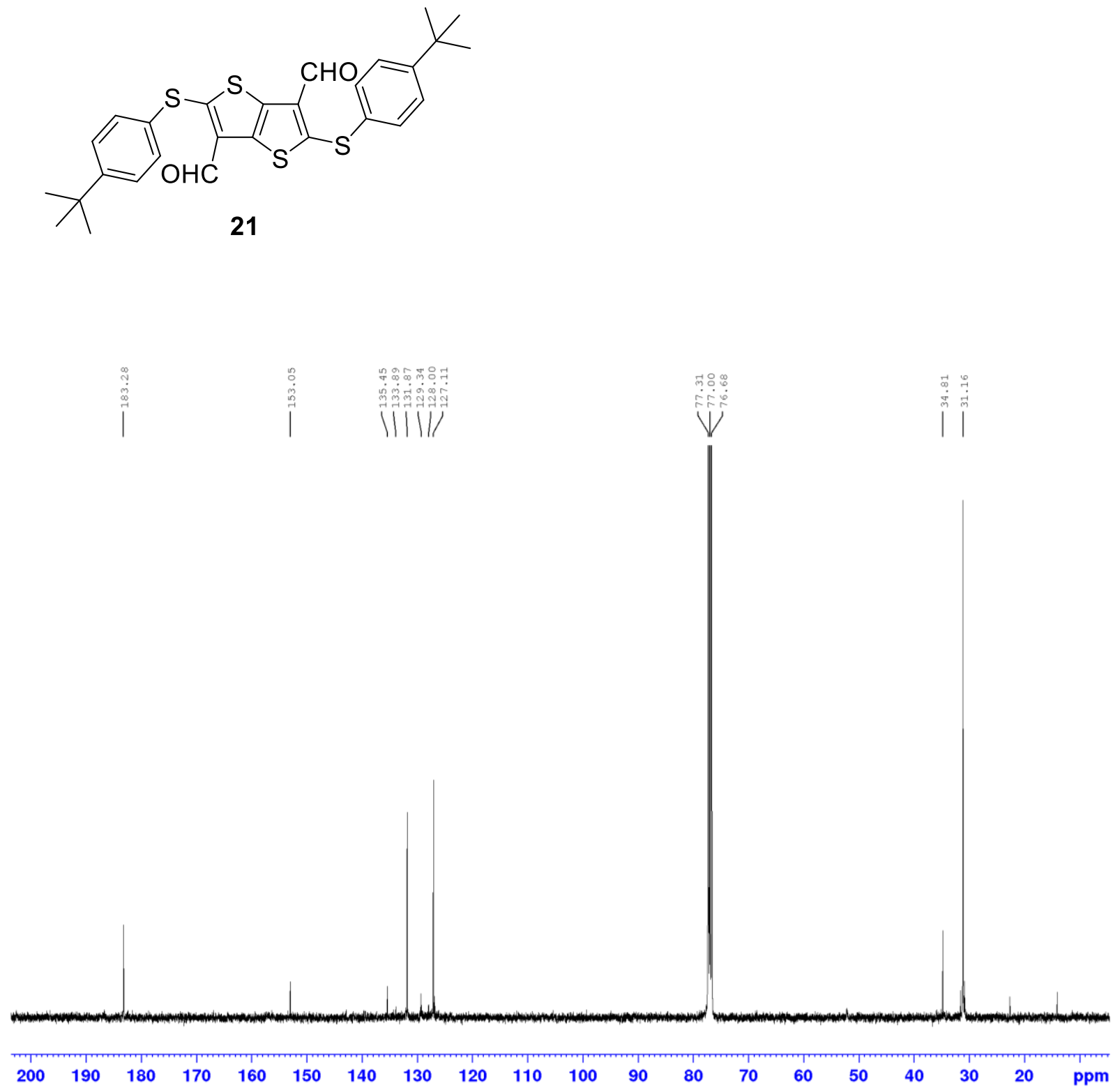
${ }^{1} \mathrm{H}$ NMR spectrum (400 MHz, $\mathrm{CDCl}_{3}, 298 \mathrm{~K}$ )
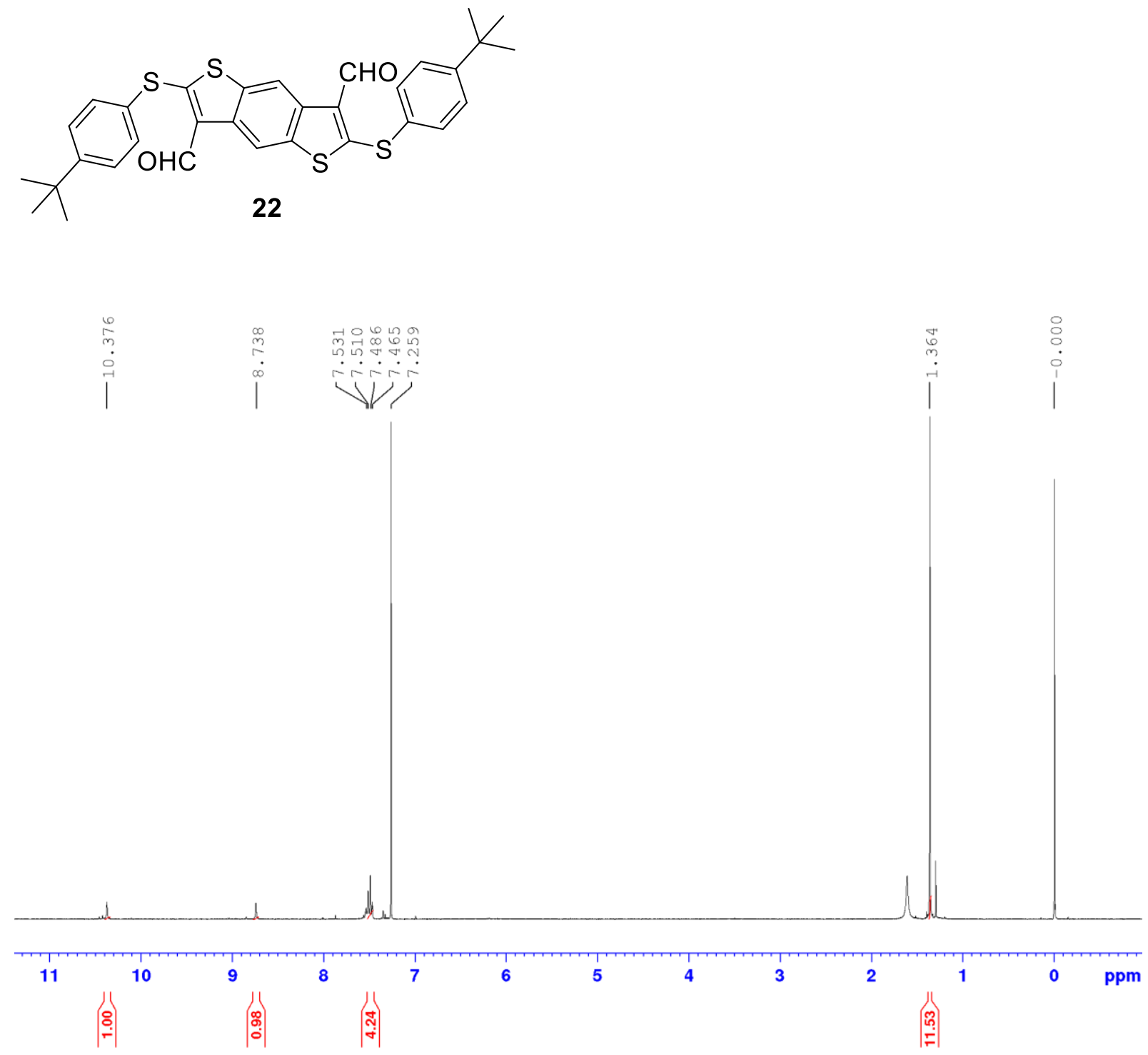
${ }^{13} \mathrm{C}\left\{{ }^{1} \mathrm{H}\right\}$ NMR spectrum (101 MHz, $\left.\mathrm{CDCl}_{3}, 298 \mathrm{~K}\right)$<smiles>Cc1c(Sc2ccc(C(C)(C)C)cc2)sc2cc3c(CO)c(Sc4ccc(C(C)(C)C)cc4)sc3cc12</smiles>

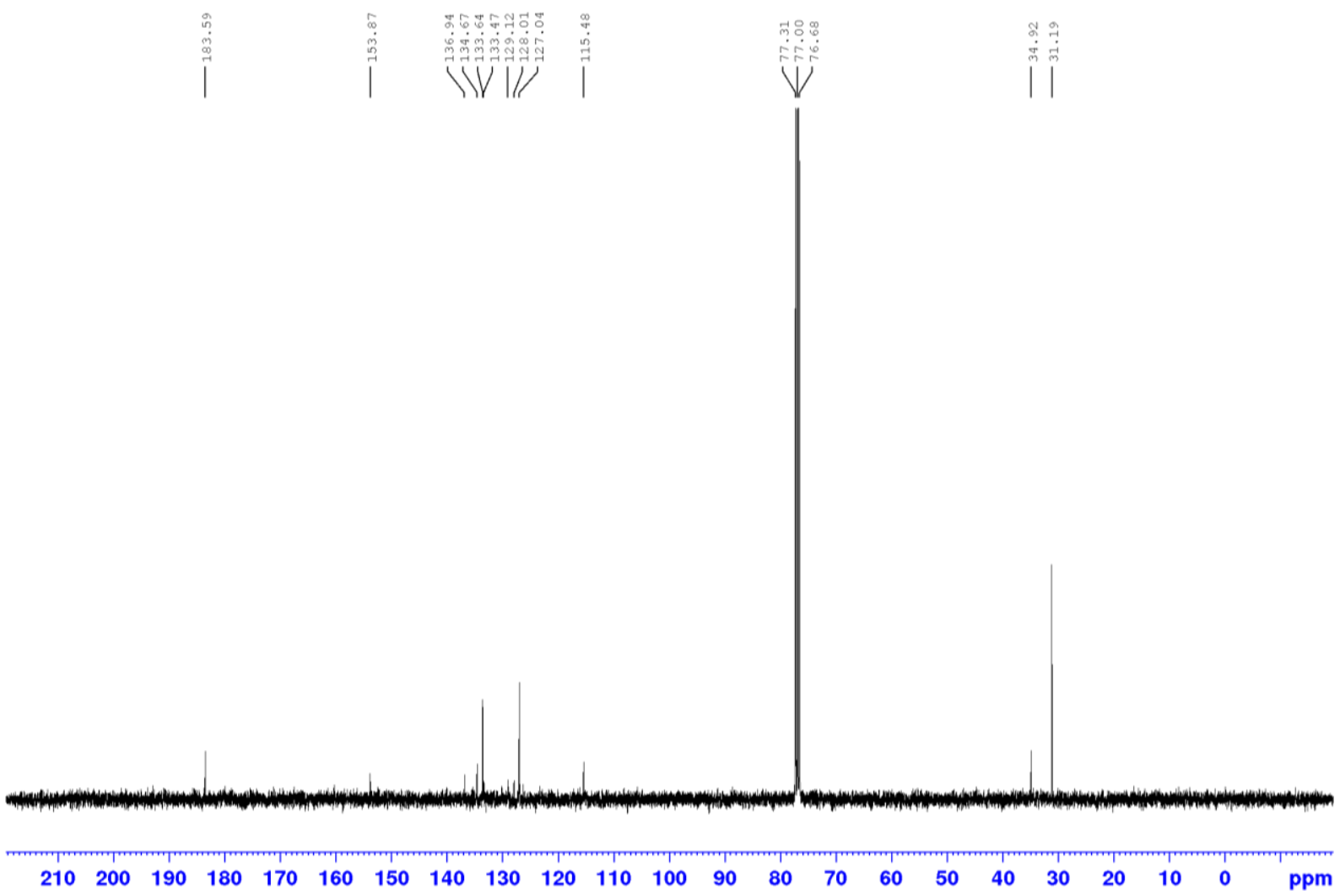


Thermogravimetric and Differential Thermal Analysis. Thermogravimetric and differential thermal analysis were performed on a Seiko Instruments SSC6300 Thermo Analyzer with a heating rate of $10 \mathrm{~K} / \mathrm{min}$ in the temperature range of $25-300{ }^{\circ} \mathrm{C}$ under an $\mathrm{N}_{2}$ atmosphere

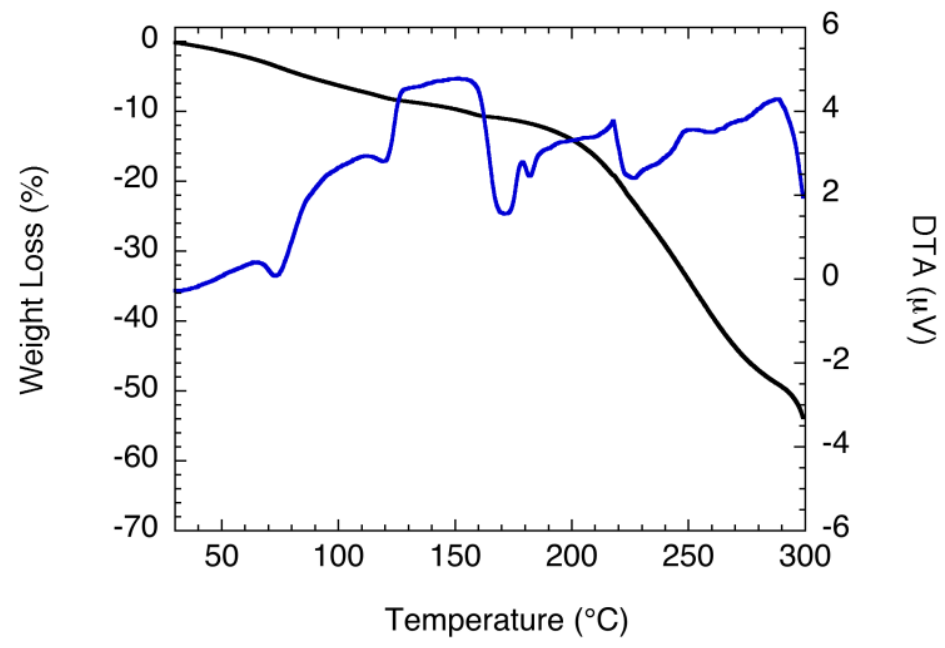

Figure S4. TGA (solid black line) and DTA (solid blue line) data for $\mathbf{1}$ under an $\mathrm{N}_{2}$ atmosphere.

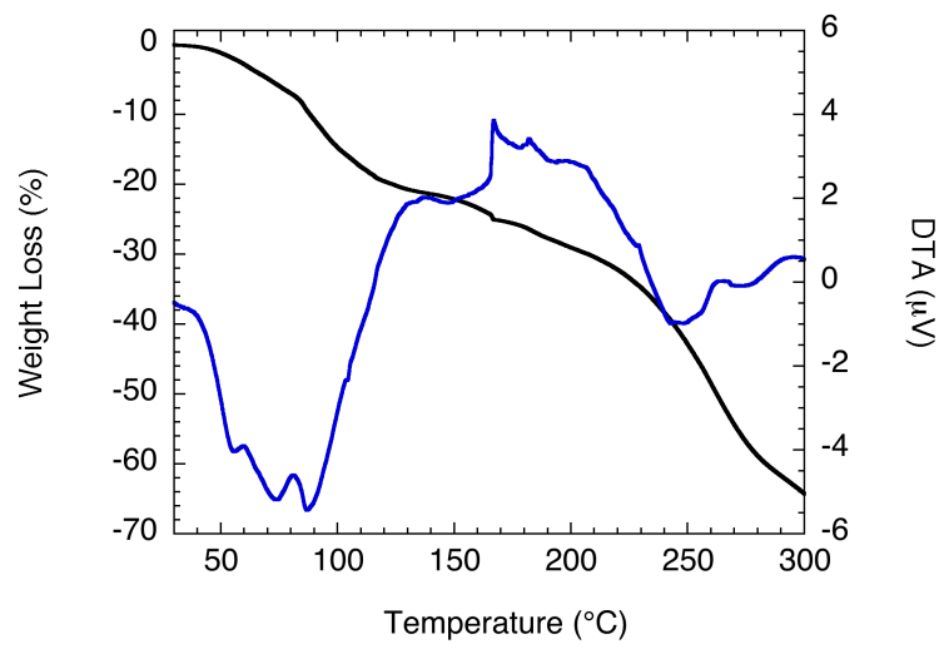

Figure S5. TGA (solid black line) and DTA (solid blue line) data for 2 under an $\mathrm{N}_{2}$ atmosphere. 


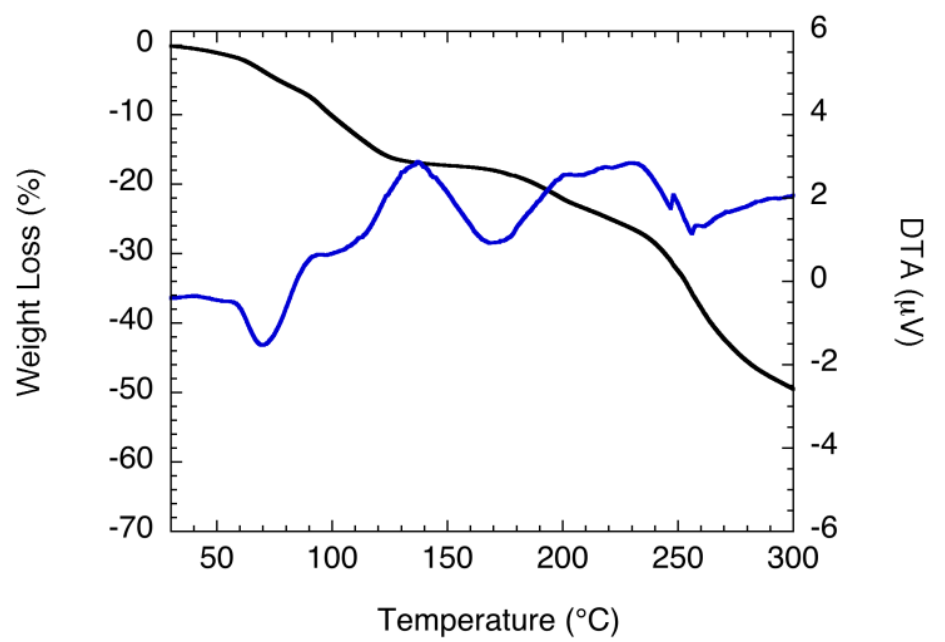

Figure S6. TGA (solid black line) and DTA (solid blue line) data for 3 under an $\mathrm{N}_{2}$ atmosphere.

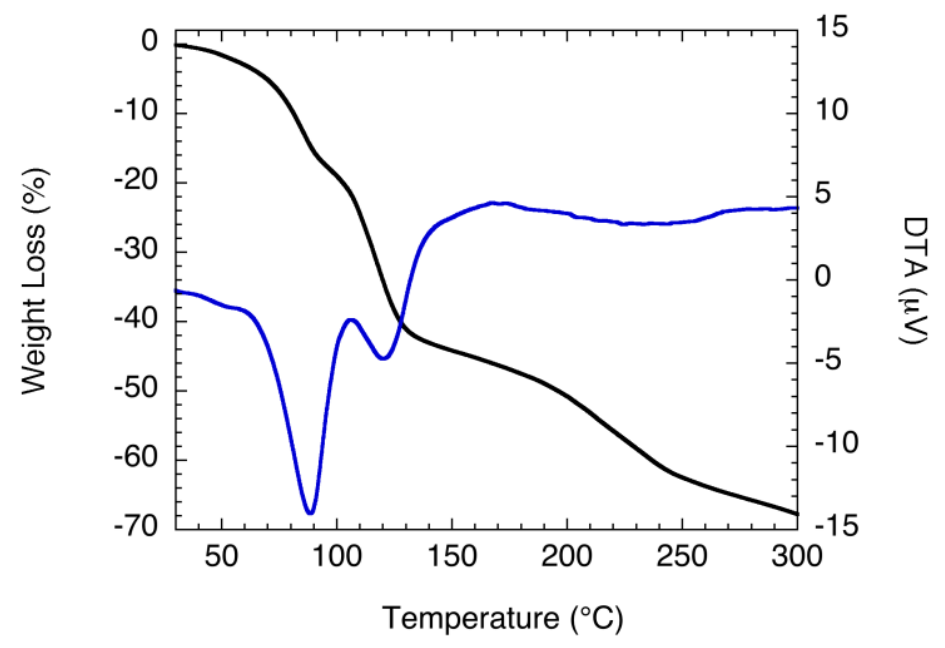

Figure S7. TGA (solid black line) and DTA (solid blue line) data for 4 under an $\mathrm{N}_{2}$ atmosphere. 


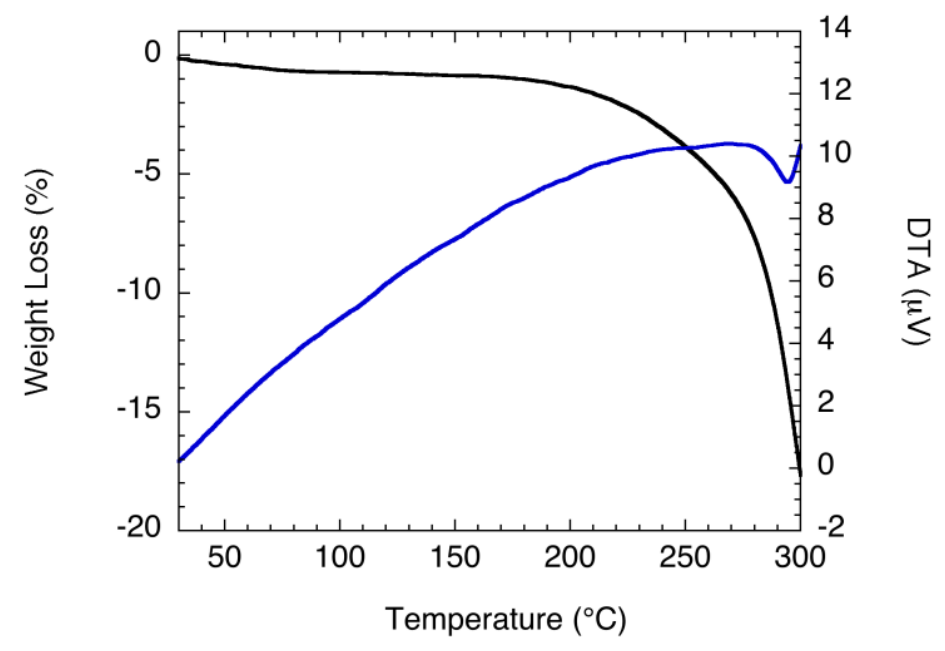

Figure S8. TGA (solid black line) and DTA (solid blue line) data for 5 under an $\mathrm{N}_{2}$ atmosphere.

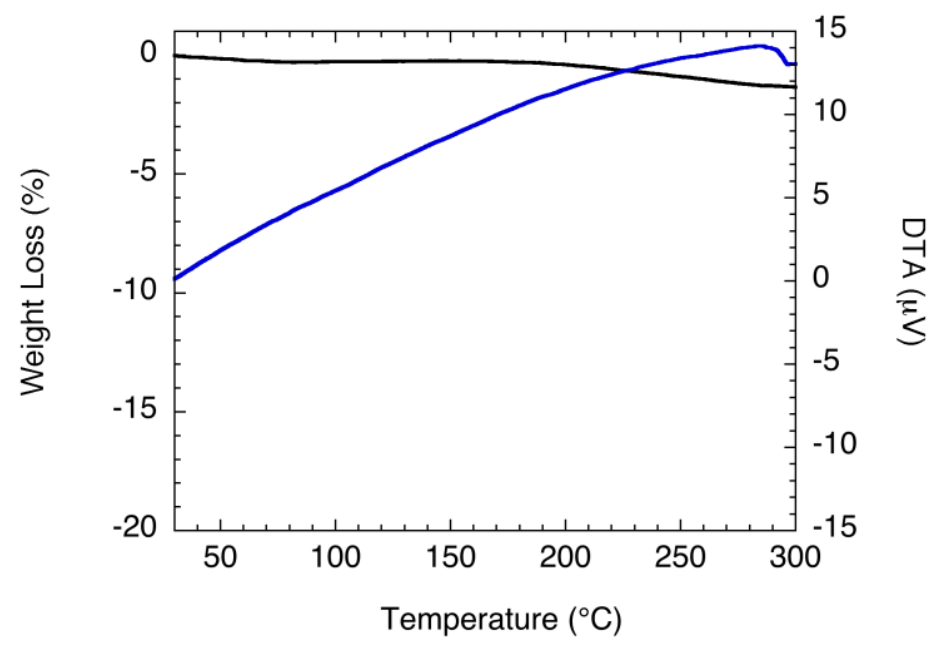

Figure S9. TGA (solid black line) and DTA (solid blue line) data for 6 under an $\mathrm{N}_{2}$ atmosphere. 


\section{References}

(1) Sheldrick, G. M. Crystal structure refinement with SHELXL. Acta Crystallogr. Sect. A 2015, 71, 3-8.

(2) Gaussian 16, Revision A.03, Frisch, M. J.; Trucks, G. W.; Schlegel, H. B.; Scuseria, G. E.; Robb, M. A.; Cheeseman, J. R.; Scalmani, G.; Barone, V.; Petersson, G. A.; Nakatsuji, H.; Li, X.; Caricato, M.; Marenich, A. V.; Bloino, J.; Janesko, B. G.; Gomperts, R.; Mennucci, B.; Hratchian, H. P.; Ortiz, J. V.; Izmaylov, A. F.; Sonnenberg, J. L.; Williams-Young, D.; Ding, F.; Lipparini, F.; Egidi, F.; Goings, J.; Peng, B.; Petrone, A.; Henderson, T.; Ranasinghe, D.; Zakrzewski, V. G.; Gao, J.; Rega, N.; Zheng, G.; Liang, W.; Hada, M.; Ehara, M.; Toyota, K.; Fukuda, R.; Hasegawa, J.; Ishida, M.; Nakajima, T.; Honda, Y.; Kitao, O.; Nakai, H.; Vreven, T.; Throssell, K.; Montgomery, Jr., J. A.; Peralta, J. E.; Ogliaro, F.; Bearpark, M. J.; Heyd, J. J.; Brothers, E. N.; Kudin, K. N.; Staroverov, V. N.; Keith, T. A.; Kobayashi, R.; Normand, J.; Raghavachari, K.; Rendell, A. P.; Burant, J. C.; Iyengar, S. S.; Tomasi, J.; Cossi, M.; Millam, J. M.; Klene, M.; Adamo, C.; Cammi, R.; Ochterski, J. W.; Martin, R. L.; Morokuma, K.; Farkas, O.; Foresman, J. B.; Fox, D. J. Gaussian, Inc., Wallingford CT, 2016.

(3) (a) Lee, C.; Yang W.; Parr, R. G. Development of the Colle-Salvetti correlation-energy formula into a functional of the electron density. Phys. Rev. B 1988, 37, 785. (b) Becke, A. D. Density-functional thermochemistry. III. The role of exact exchange. J. Chem. Phys. 1993, 98, 5648. (c) Stephens, P. J.; Devlin, F. J.; Chabalowski, C. F.; Frisch, M. J. Ab initio calculation of vibrational absorption and circular dichroism spectra using density functional force fields. J. Phys. Chem. 1994, 98, 11623. 